Mens

Revue d'histoire intellectuelle et culturelle

mens

\title{
The Surrender of Montreal to General Amherst de Francis Hayman et l'identité impériale britannique
}

\section{Laurent Turcot}

Volume 12, numéro 1, automne 2011

URI : https://id.erudit.org/iderudit/1010567ar

DOI : https://doi.org/10.7202/1010567ar

Aller au sommaire du numéro

Éditeur(s)

Centre de recherche en civilisation canadienne-française

ISSN

1492-8647 (imprimé)

1927-9299 (numérique)

Découvrir la revue

Citer cet article

Turcot, L. (2011). The Surrender of Montreal to General Amherst de Francis Hayman et l'identité impériale britannique. Mens, 12(1), 91-135.

https://doi.org/10.7202/1010567ar
Résumé de l'article

Dans le présent article, j'analyserai le tableau The Surrender of Montreal to General Amherst de Francis Hayman, qui représente une victoire de l'Empire britannique sur la France en terre américaine en 1760. Mon objectif est de comprendre les origines de l'oeuvre commandée par Jonathan Tyers, propriétaire du Vauxhall à Londres, où est présenté le tableau, et de saisir ce qu'on a voulu transmettre par cette oeuvre exposée dans un contexte bien particulier, soit un espace de loisir. J'examinerai aussi de quelle façon elle a permis de raconter à un public donné les événements entourant la prise du Canada dans les années 1760 . La manière de raconter et de représenter la conquête du Canada se rattache, certes, à l'idée de grandeur de l'Empire, mais aussi, et surtout, à celle qui consiste à montrer que l'Angleterre devient la seule grande puissance européenne (supériorité) à pouvoir soutenir des populations coloniales par des valeurs typiquement britanniques d'humanité, de clémence et de charité, mais qui ont également une portée universelle. Ces valeurs permettent d’imposer et de légitimer la Pax Britannica en Amérique. 


\title{
The Surrender of Montreal to General Amberst de Francis Hayman et l'identité impériale britannique ${ }^{1}$
}

\author{
Laurent Turcot \\ Département d'histoire \\ Université du Québec à Trois-Rivières
}

\section{Résumé}

Dans le présent article, j'analyserai le tableau The Surrender of Montreal to General Amberst de Francis Hayman, qui représente une victoire de l'Empire britannique sur la France en terre américaine en 1760. Mon objectif est de comprendre les origines de l'œuvre commandée par Jonathan Tyers, propriétaire du Vauxhall à Londres, où est présenté le tableau, et de saisir ce qu'on a voulu transmettre par cette œuvre exposée dans un contexte bien particulier, soit un espace de loisir. J'examinerai aussi de quelle façon elle a permis de raconter à un public donné les événements entourant la prise du Canada dans les années 1760. La manière de raconter et de représenter la conquête du Canada se rattache, certes, à l'idée de grandeur de l'Empire, mais aussi, et surtout, à celle qui consiste à montrer que l'Angleterre devient la seule grande puissance européenne (supériorité) à pouvoir soutenir des populations coloniales par des valeurs typiquement britanniques d'humanité, de clémence et de charité, mais qui ont également une portée universelle. Ces valeurs permettent d'imposer et de légitimer la Pax Britannica en Amérique.

\footnotetext{
${ }^{1}$ L'auteur tient à remercier les lecteurs anonymes pour leurs remarques sur la première version de ce texte.
} 


\section{Abstract}

In this article, I examine a painting representing the 1760 victory of the British Empire over France in North America, namely The Surrender of Montreal to General Amherst by Francis Hayman. I seek to understand the origins of this piece ordered by Jonathan Tyers, owner of London's Vauxhall, the recreational space where The Surrender of Montreal was exhibited, and to understand the meaning that it sought to convey to the public regarding the events surrounding the fall of New France. The piece sought to convey a strong message regarding the apparent greatness of the British Empire, but it also sought to show that Britain had emerged as Europe's only truly great power; the only power able to govern colonial populations through the universal - and typically British - values of humanity, clemency, and charity. These values allowed Britain to impose and legitimise the "Pax Britannica" in North America.

L'historiographie portant sur l'émergence de l'identité impériale britannique est riche. Depuis Linda Colley et son Britons: Forging the Nation 1707-1837, nombre d'historiens ont repris l'idée que les Britanniques du long XVIII ${ }^{\mathrm{e}}$ siècle "defined themselves as Protestants struggling for survival against the world's foremost Catholic power. They defined themselves against the French as they imagined them to be, superstitious, militarist, decadent and unfree ${ }^{2}$ ". La France devient ainsi l'altérité signifiante de la définition identitaire impériale britannique. Cette interprétation, aussi séduisante et convaincante qu'elle puisse paraître, laisse de côté non seulement tout un pan de la culture britannique, soit les échanges franco-britanniques ${ }^{3}$, mais également

\footnotetext{
${ }^{2}$ Linda Colley, Britons: Forging the Nation 1707-1837, New Haven, Yale University Press, 1992, p. 6, et Captives: Britain, Empire and the World 1600-1850, New York, Anchor Books, 2004. Voir également Anthony D. Smith, "The Origins of Nations ", Ethnic and Racial Studies, vol. 12, n 3 (1989), p. 340-367.

${ }^{3}$ Cet aspect est de plus en plus étudié. L'histoire comparative et, plus spécifiquement, la notion de transfert culturel montrent que la réalité est plus complexe. Voir notamment François-Joseph Ruggiu, Lindividu et la famille dans les sociétés urbaines
} 
l'intégration des colonies conquises à la suite de la guerre de Sept Ans. Plusieurs historiens ont offert des études déterminantes pour le cas des 13 colonies américaines ${ }^{4}$, mais la question canadienne $e^{5}$ est quelque peu restée dans l'ombre de cette histoire culturelle. C'est sur ce sujet que j'entends apporter un éclairage supplémentaire. Chez Colley, le Canada n'est mentionné qu'à trois reprises, en plus de quelques références éparses qui ne portent que sur les événements de la guerre de Sept Ans. Qu'en est-il de la place que l'on confere à cette nouvelle colonie dans la définition de l'identité impériale?

Philip Lawson ${ }^{6}$, dans The Imperial Challenge, analyse la polarisation de l'opinion publique britannique après la conquête du Canada, principalement à partir des journaux britanniques de l'époque. À terme, Lawson établit les bases des débats ayant mené à l'Acte de Québec (1774). Cet aspect ne permet pas nécessairement de saisir la

anglaise et française (1720-1780), Paris, PUPS, 2007; Sophie Audidière, Simon Burrows, Edmond Dziembowski et Ann Thomson (dir.), Cultural Transfers: France and Britain in the Long Eighteenth Century, Oxford, Voltaire Foundation, 2010; et Leora Auslander, Des révolutions culturelles : la politique du quotidien en GrandeBretagne, en Amérique et en France, XVII -XIXe siècles, Toulouse, Presses universitaires du Mirail, 2010.

${ }^{4}$ Holger Hoock, Empires of the Imagination: Politics, War, and the Arts in the British World, 1750-1850, London, Profile, 2010; Eliga H. Gould, The Persistance of Empire: British Political Culture in the Age of the American Revolution, Chapel Hill, University of North Carolina Press, 2000 ; Peter James Marshall, The Making and Unmaking of Empires: Britain, India, and America, c. 1750-1783, Oxford, Oxford University Press, 2005; Christine Daniels et Michael V. Kennedy (dir.), Negotiated Empires: Centers and Peripheries in the Americas, 1500-1820, New York, Routledge, 2002; Eran Shalev, "Empire Transformed: Britain in the American Classical Imagination, 1758-1783 ", Early American Studies, vol. 4, n 1 (2006), p. 112-146; et Douglas Fordham, British Art and the Seven Years' War: Allegiance and Autonomy, Philadelphia, University of Pennsylvania Press, 2010.

${ }^{5}$ Voir notamment Elizabeth Mancke, «Another British America: A Canadian Model for the Early modern British Empire ", The Journal of Imperial and Commonwealth History, vol. 25, no 1 (1997), p. 1-36.

${ }^{6}$ Philip Lawson, The Imperial Challenge: Quebec and Britain in the Age of the American Revolution, Montréal, McGill-Queen's University Press, 1990; et Joan Coutu, Persuasion and Propaganda: Monuments and the Eighteenth-Century British Empire, Montréal, McGill-Queen’s University Press, 2006. 
pleine mesure de l'intégration du Canada à l'identité impériale. Pour John E. Crowley, dans Imperial Landscapes, il faut saisir cette transformation de l'espace colonial britannique au XVIII ${ }^{\mathrm{e}}$ siècle en croisant les approches politiques, mais surtout en tenant compte de la culture visuelle qui a permis l'apparition de référents culturels communs ${ }^{7}$. L'idée est de comprendre comment " the diplomatic aftermath of the Seven Years' War presented Britons with the prospect of a global territorial empire ruling non-British people ${ }^{8}$. Crowley rappelle à cet effet qu'il est nécessaire de considérer la peinture comme un "visual message [...] about a landscape whose scenic attractions and rustic tranquillity mitigated its appropriation by conquest ${ }^{9}$ ", idée déjà évoquée par Colin $\mathrm{M}$. Coates quand il affirme, à propos des récits de voyage de cette période, que les "Europeans invoked aesthetic principles in order to establish dominion over foreign territories. [...] Travel and exploration literature addressed new landscapes in Old World terms, if only to make sense of them to the home audience for which they were written $n^{10} "$. On livre alors une image du Canada qui "helped both colonists and metropolitans to maintain their identity and self-respect as civilized and civilizing Britons. [...] As eighteenth-century British artists increasingly took an interest in their country's scenery, they adapted the idealizing style of the picturesque for their topographic representations ${ }^{11}$ ". Cette tendance suit celle qui est lancée en Angleterre au milieu du siècle, faisant du paysage un élément constitutif de l'identité de l'Empire :

\footnotetext{
${ }^{7}$ John E. Crowley, Imperial Landscapes: Britain's Global Visual Culture, 1745-1820, New Haven, Yale University Press, 2011.

${ }^{8}$ Ibid., p. 50.

9 John E. Crowley, " "Taken on the Spot”: The Visual Appropriation of New France for the Global British Landscape ", Canadian Historical Review, vol. 86, ${ }^{\circ} 1$ (mars 2005), p. 1.

${ }^{10}$ Colin M. Coates, "Like "The Thames towards Putney": The Appropriation of Landscape in Lower Canada ", Canadian Historical Review, vol. 74, n 3 (1993), p. 317. Voir également Les transformations du paysage et de la société au Québec sous le régime seigneurial, Sillery, Septentrion, 2003, et Mary Louise Pratt, Imperial Eyes: Travel Writing and Transculturation, London, Routledge, 1992.

${ }^{11}$ Crowley, " "Taken on the Spot": The Visual Appropriation of New France », p. 3-4.
} 
"Imperial landscape art linked three crucial developments in eighteenthcentury British culture and politics: 1) the creation of a British identity [...], 2) the imperial assertion of this identity through commercial expansion and strategic success, and 3) the commodified representation of these identities and successes to a viewing public ${ }^{12}$."

Joan Coutu soutient que les différents monuments qui sont érigés après la guerre de Sept Ans sont autant de «baromètres de l'impérialisme " que l'on a longtemps limités à la presse écrite pour comprendre " the growth of cultural and imperial infrastructures in Britain and its colonies ${ }^{13}$ ». La conquête du Canada est également à mettre en relation avec la constitution de l'Empire atlantique britannique $^{14}$ qui tâche de maintenir sa cohésion par tous les moyens. Kathleen Wilson va plus loin en soutenant que se mettent en place, au milieu du XVIII" siècle, des "politics of identity which celebrated the English nation for its freedom, religion, prosperity and imperial standing as well as for the patriotic and martial spirit of its inhabitants". D'ailleurs la victoire sur la France en 1763 " was clearly the fulfilment and ultimate expression of the mercantilist-imperialist goals and aspirations articulated for the past three years ${ }^{15}$ " visant à toucher toutes les catégories sociales ${ }^{16}$.

Pour David Solkin, la Grande-Bretagne et, plus spécifiquement, les élites londoniennes sont marquées par ce qu’il qualifie de «Exhibitions of Sympathy ", soit l'idée que la peinture "was shortly to assume a prominent public role in the blossoming culture of sentiment». L'identité britannique, du moins celle que l'on retrouve dans la peinture et les textes de l'époque, notamment ceux de David Hume ou de Samuel

${ }^{12}$ Ibid.

${ }^{13}$ Coutu, Persuasion and Propaganda, p. 7.

${ }^{14}$ Voir, entre autres, John H. Elliott, Empires of the Atlantic World: Britain and Spain in America 1492-1830, New Haven, Yale University Press, 2007; Colin Kidd, British Identities Before Nationalism: Ethnicity and Nationhood in the Atlantic World, 1600-1800, New York, Cambridge University Press, 1999.

${ }^{15}$ Kathleen Wilson, The Sense of the People: Politics, Culture and Imperialism in England, 1715-1785, Cambridge, Cambridge University Press, 1995, p. 173 et 193.

${ }^{16}$ Voir également Gerald Newman, The Rise of English Nationalism: A Cultural History, 1740-1830, London, Palgrave Macmillan, 1987, p. 217. 
Richardson, ne s'articule pas nécessairement sur l'opposition avec la France, mais sur la glorification d'un "discursive universe of mideighteenth-century "humanity", alongside the hospitals, theaters, and other site of polite assembly dedicated to the cultivation of a sympathetic spectatorship ${ }^{17}$ ". Martin Myrone, de son côté, montre comment la seconde moitié du XVIII ${ }^{\mathrm{e}}$ siècle voit se définir un ensemble de caractéristiques culturelles visant à décrire et à montrer ce qu'est le héros contemporain avec, au cœur de ce changement, la prise en charge de la masculinité par des peintres comme Benjamin West, Alexander Runciman et James Barry ${ }^{18}$.

En m'inscrivant dans la voie tracée par Crowley et Solkin, je me propose d'étudier la capitulation de Montréal, en fait, la représentation qu'en a faite un peintre, afin de comprendre le contexte qui entoure cette œuvre, les références qu'on y retrouve et les interprétations des contemporains. La culture visuelle britannique est plurielle, elle ne peut se définir que par un seul aspect, qu'il s'agisse de l'identité de l'Empire qui se fait en opposition à la France (Colley) ou encore de la représentation rustique d'un territoire (Crowley). Au midi du siècle, et ce sera là un des apports de ce texte, la notion de supériorité britannique est promue, et ce, bien avant la victoire finale de 1763 que constitue le traité de Paris. Du siège de Louisbourg en passant par les plaines d'Abraham pour en arriver à la capitulation de Montréal, la guerre de Sept Ans voit l'armée britannique gagner sur presque tous les fronts; l'heure est aux célébrations. Pourtant, une question demeure : quels sont les vecteurs qui permettent de rendre les choses intelligibles aux contemporains, de les renseigner sur les mouvements de troupes et de leur faire connaître les régions dans lesquelles se déroulent les batailles?

${ }^{17}$ David Solkin, Painting for Money: The Visual Arts and the Public Sphere in EighteenthCentury England, New Haven, Yale University Press, 1993, p. 157 et 199.

${ }^{18}$ Martin Myrone, Bodybuilding: Reforming Masculinities in British Art, 1750-1810,

New Haven, Yale University Press, 2005, p. 33-120. 
L'analyse que je propose aujourd'hui montre une des formes d'appropriation de cette identité qui se définit au milieu du siècle. La manière de raconter et de montrer la conquête du Canada se rattache, certes, à l'idée de grandeur de l'Empire, mais aussi, et surtout, à celle qui consiste à montrer que l'Angleterre devient la seule grande puissance européenne (supériorité) à pouvoir soutenir des populations coloniales par des valeurs typiquement britanniques d'humanité, de clémence et de charité, mais qui ont également une portée universelle. Ces valeurs permettent d'imposer et de légitimer la Pax Britannica en Amérique. Avant même la signature du traité de Paris (1763), donc de la fin de la guerre de Sept Ans, et c'est là un autre point que j'entends exposer, l'architecture culturelle visant à légitimer la conquête du Canada est déjà en place. Elle fait également l'objet d'une appropriation de la part d'une frange de la population, l'élite politique, économique et culturelle, qui espère capitaliser sur l'intérêt qu'on porte à la grandeur et à la gloire britannique en Amérique.

Le fameux Death of General Wolfe peint en 1771 par Benjamin West sert, la plupart du temps, à illustrer une des batailles marquantes de la guerre de Sept Ans. Pourquoi est-ce ce tableau qui a été retenu pour illustrer la victoire de la campagne canadienne? Plusieurs spécialistes $^{19}$ ont analysé l'œuvre de West en expliquant pourquoi on s'est emparé, en Angleterre comme au Canada, du tableau pour en faire la représentation parfaite du conflit. Cependant, d'autres peintres, contemporains de West, se sont attelés à la tâche de représenter la victoire anglaise en Amérique; Francis Hayman est un de ceux-là et il l'a fait dès le début de l'année 1761, soit quelques mois après la

${ }^{19}$ Voir notamment Anne U. Abrams, The Valiant Hero: Benjamin West and GrandStyle History Painting, Washington, Smithsonian Institution Press, 1985; Stephen Brumwell, Paths of Glory: the Life and Death of General Wolfe, Montréal, McGillQueen's University Press, 2006; Vivien Green Fryd, "Rereading the Indian in Benjamin West's Death of General Wolfe ", American Art, vol. 9, n 1 (printemps 1995), p. 73-85; et Dennis Montaga, "Benjamin West's The Death of General Wolfe: A Nationalist Narrative ", American Art Journal, vol. 13, n 2 (printemps 1981), p. 72-88. 
capitulation de Montréal. L'œuvre exposée au Vauxhall servira ici de porte d'entrée dans le quotidien politique au midi du siècle.

\section{Montréal au Vauxhall}

Dans A Description of Vaux-hall Gardens, Being a Proper Companion and Guide for All Who Visit that Place, publié en 1762, l'auteur, anonyme, expose au voyageur ce qu'il peut observer et apprécier dans ce jardin intérieur londonien où l'on vient se récréer en écoutant de la musique, en dansant ou en achetant quelques rafraîchissements le long des allées bordées d'arbres ${ }^{20}$. Dans la rotonde du Vauxhall, entre les quatre colonnes soutenant la voûte de l'établissement, l'auteur signale de grands tableaux, dont la taille moyenne est de quatre mètres sur cinq : "Mr. Hayman was employed to celebrate with his masterly pencil, some of the most glorious transactions of the present war, and in the year 1761, the first picture was exhibited to view. It represents the surrender of Montreal in Canada, to the victorious arms of Great Britain, commanded by general Amberst ${ }^{21}$."

Brian Allen, spécialiste de l'œuvre peinte de Hayman, souligne que " [a] lthough none of the four original pictures which were painted between 1761 and 1764 survives, with the aid of an engraving made after one of them, modelli for two of the others and a lengthy printed description of the forth picture, it is possible to reconstruct a fairly accurate idea of their appearance ${ }^{22}$ ". À côté du tableau sur Amherst, il était également possible d'apprécier The Triumph on Britannia, Lord Clive meeting with Mir Jaffar after the Battle of Plassey, peints tous les deux en 1762 et, enfin, en 1764, Britannia distributing Laurels to the

${ }^{20}$ Jonathan Conlin, "Vauxhall on the Boulevard: Pleasure Gardens in London and Paris, 1764-1784 ", Urban History, vol. 35, n 1 (2008), p. 24-47; David Coke et Alan Borg, Vauxhall Gardens: A History, New Haven, Yale University Press, 2011.

${ }^{21}$ A Description of Vaux-hall Gardens, Being a Proper Companion and Guide for All Who Visit that Place, illustrated with Copper-Plates, London, S. Hooper, 1762, p. 24.

${ }^{22}$ Brian Allen, "Rule Britannia? History Painting in 18th-Century Britain ", History Today, vol. 45, no 6 (juin 1995), p. 15. Voir également Francis Hayman, New Haven, Yale University Press, 1987. 
Victorious Generals, tous les trois réalisés par Hayman. La volonté de chanter les gloires coloniales est ici assez évidente, mais encore fautil contextualiser l'œuvre en question. Une première recherche a permis de déterminer le lieu où est conservé le modèle de la peinture du général Amherst : la Beaverbrook Art Gallery à Fredericton. Il est apparu que le tableau ne portait pas un, mais trois titres: Clemency of General Amherst, The Humanity of General Amberst et The Surrender of Montreal to General Amberst. Le deuxième modèle de ce tableau a été acquis par le Musée canadien de la guerre à Ottawa en $1995^{23}$. Il s'intitule The Charity of General Amherst. Nous voici donc avec une œuvre qui porte quatre titres. J'aurai l'occasion de revenir sur ces dénominations.

Il serait faux d'affirmer que cette représentation de Francis Hayman est inconnue. Les historiens de l'art et ceux de la guerre l'ont signalée, soit en l'intégrant dans le renouveau de la peinture d'histoire en Angleterre au XVIII ${ }^{\mathrm{e}}$ siècle, soit pour illustrer la dernière grande victoire de la conquête du Canada, mais, dans les deux cas, sans réellement insister spécifiquement sur la nature et la portée de l'œuvre ${ }^{24}$. La place de l'image dans l'histoire fait l'objet de nombreuses interrogations de la part des historiens ${ }^{25}$. Si certains ne s'en servent que comme moyen d'illustrer des événements, d'autres font d'elle

${ }^{23}$ Laura Brandon, "Francis Hayman's The Charity of General Amherst: A New Acquisition for the Canadian War Museum ", The Journal of Canadian Military History, vol. 3, n 2 (1994), p. 111-112. Voir également Lawrence Gowing, "Hogarth, Hayman, and the Vauxhall Decorations ", The Burlington Magazine, vol. 95, nº 598 (1959), p. 4-19.

${ }^{24}$ Miles Ogborn, Spaces of Modernity: London's Geographies 1680-1780, New York, The Guilford Press, 1998; Peter de Bolla, The Education of the Eye: Painting, Landscape, and Architecture in Eighteenth-Century Britain, Stanford, Stanford University Press, 2003; Fred Anderson, Crucible of War: The Seven Years' War and the Fate of Empire in British North America 1754-1766, New York, Vintage Books, 2000 ; Joseph Burke, English Art 1714-1800, Oxford, Clarendon Press, 1976; et R. Scott Stephenson, Clash of Empires: the British, French \& Indian War, 17541763, Pittsburgh, Historical Society of Western Pennsylvania, 2005.

${ }^{25}$ Christian Delporte, Laurent Gervereau et Denis Maréchal (dir.), Quelle est la place des images en histoire?, Paris, Nouveau Monde, 2008; Annie Duprat, Images et histoire: outils et méthodes d'analyse des documents iconographiques, Paris, Belin, 2007. 
une source à part entière, avec ses limites, ses constructions et ses reconstructions, sa portée limitée, etc. C'est dans cette seconde voie que s'inscrit la présente analyse. Comme l'évoque Simon Schama, l'art a une puissance qui permet à l'artiste de susciter un "drumbeating patriotism ${ }^{26}$ ». Peter Harrington signale la grande diffusion des ouvres des peintres britanniques au XVIII ${ }^{\mathrm{e}}$ siècle $^{27}$, diffusion qui renforce la constitution d'un état d'esprit commun et d'une culture visuelle répandue et critique. L'art devient un moyen de se renseigner, mais de manière bien singulière, car "for them [the people in England] war was a distant event and their frames of reference came from the odd print or engraving which they saw hanging in printshops, taverns and coffee houses $^{28}$ ". Il s'agit donc d'amener le Canada dans le quotidien des Britanniques.

Ami de William Hogarth et de l'acteur David Garrick, Francis Hayman enseigne à Thomas Gainsborough, en plus d'illustrer nombre d'ouvrages comme les romans de Richardson ou, encore, des éditions contemporaines de William Shakespeare, ce qui place l'artiste au cœur des grands mouvements artistiques qui définissent son époque ${ }^{29}$. Il est également un proche de la famille Tyers, dont il peindra le portrait en 1740 : Jonathan Tyers and his family. S'inspirant des jardins publics et des autres Spring Gardens londoniens, Jonathan Tyers, en 1728 , met sur pied un jardin de plaisir, nommé Vauxhall, pour lequel les visiteurs doivent payer des droits d'entrée. Le public profite ainsi

\footnotetext{
${ }^{26}$ Simon Schama, Landscape and Memory, New York, Vintage Books, 1996, p. 357.

${ }^{27}$ Voir Crowley, " "Taken on the Spot": The Visual Appropriation of New France for the Global British Landscape "; Coates, "Like "The Thames towards Putney": The Appropriation of Landscape in Lower Canada "; Beth Fowkes Tobin, Picturing Imperial Power: Colonial Subjects in Eighteenth-Century British Painting, Durham, Duke University Press, 1999; Nathalie Miglioli et PierreOlivier Ouellet (dir.), Mélanges sur l'art au Québec historique (XVII'-XIX siècles), Centre de recherche interuniversitaire sur la littérature et la culture québécoises, 2009, coll. "Interlignes ".

${ }^{28}$ Peter Harrington, British Artists and War: The Face of Battle in Paintings and Prints, 1700-1914, London, Greenhill, 1993, p. 25.

${ }^{29}$ Allen, Francis Hayman, p. 3.
} 
d'allées aménagées, d'orchestres, de salles richement décorées, bref, le Vauxhall est une sorte de cour en plein air dont l'admission coûte un shilling. Il s'agit donc d'un public provenant majoritairement de classes sociales supérieures (noblesse et haute bourgeoisie), quoique l'on trouve parfois le commun qui se paie un petit luxe. Le Vauxhall, forme de loisir typiquement britannique, dessille rapidement les yeux des Londoniens et bientôt de toute l'Europe ${ }^{30}$.

Tyers offre ainsi un espace de loisir dans lequel il est de bon ton de voir et d'être vu. À cette fonction, il ajoute un déterminant évident qui consiste à capitaliser sur les récréations offertes au public londonien. Il ne convient pas d'offrir uniquement des plaisirs futiles et passagers, il faut faire vibrer l'âme et la fibre nationale de l'identité britannique, cela bien sûr afin d'encourager la dépense. Exploiter l'actualité et susciter la fierté patriotique, voilà ce qui permet d'assurer l'intérêt constant et le renouvellement du public. Les sujets des œuvres exposées au Vauxhall dépassent le cadre ludique. Ils permettent d'analyser la permanence et l'évolution de l'identité britannique et, bientôt, de l'Empire, incluant les Canadiens.

Jonathan Tyers va utiliser les talents de Francis Hayman pour donner à son établissement un caractère typiquement anglais en chantant les gloires passées et, bientôt, présentes. Tyers commande ainsi plusieurs tableaux à Hayman pour décorer le Vauxhall. Des représentations des pièces historiques de Shakespeare qui toutes sont des modèles et des leçons pour le promeneur, par exemple des images représentant le Roi Lear, Hamlet, Henri Vet La Tempête. Ces œuvres sont de véritables réflexions sur le pouvoir, sa légitimité, ses responsabilités, sa fragilité et sa vanité. Pour Henri $V$, on ne manque pas de représenter la bataille d'Azincourt, grande victoire anglaise sur la France, ce qui ne va pas sans rappeler l'idée de Linda Colley et Gerald Newman selon laquelle la France serait considérée comme altérité signifiante dans le processus de définition de l'identité britannique.

${ }^{30}$ A Description of Vaux-hall Gardens, p. 21. 
Tyers entend aller plus loin. Il lui faut être au fait des dernières tendances et saisir les événements de l'actualité politique et militaire afin de donner à voir la puissance de l'Angleterre ${ }^{31}$. Dès le début de la guerre de Sept Ans, Tyers commande à John Lockman des chansons qui seront entonnées au Vauxhall. Ces dernières racontent les exploits britanniques outre-mer. Elles sont ensuite publiées, peu de temps après, dans des recueils et accessibles à tout un chacun. En 1758, Lockman propose Cape Breton and Cherburg, chanson dans laquelle, sur l'air de God Save Our Noble King, le général Amherst est comparé à Edward III ${ }^{32}$. Le vaste répertoire des chansons du Vauxhall ${ }^{33}$ montre la facture très contemporaine des thèmes choisis. Les premières batailles sur le continent américain et bientôt européen entrent rapidement dans le quotidien des populations. Comme le souligne Peter de Bolla : "Tyers was, of course, exploiting the situation of the war but we should pause to note that they were in fact commissioned before the war had ended and therefore before it had been won ${ }^{34}$."

La campagne en Amérique du Nord est un des points majeurs de la guerre de Sept Ans. La conquête du Canada est relayée par les différents journaux anglais, qui préparent la population anglaise à la victoire en chantant les gloires de ses généraux. Philip Lawson a bien montré comment la conquête du Canada est racontée en Angleterre : "The nation held its breath while news of the action on Quebec trickled across the Atlantic ${ }^{35}$." Ici et là, dans les différentes éditions des journaux, par exemple dans le British Magazine, se retrouvent, avant

\footnotetext{
${ }^{31}$ Allen, Francis Hayman, p. 62.

32 Ogborn, Spaces of Modernity, p. 143.

${ }^{33}$ The first part of the Vaux-Hall concert containing all the songs sung this season at Vaux-Hall, London, 1760; The second part of the Vaux-Hall concert being a collection of the best songs in vogue, London, printed and sold upon Snow-Hill, 1760; The complete English songster; being a collection of the newest songs that have been sung at the play-houses; also, Vauxhall, Ranelaigh, and Marybone gardens: with a great many new prologues and epilogues, medleys, London, printed for the booksellers, 1762.

${ }^{34}$ de Bolla, The Education of the Eye, p. 95.

${ }^{35}$ Lawson, The Imperial Challenge, p. 3.
} 
même les premières victoires, des descriptions du Canada, de son histoire ou encore des explications sur les mœurs des habitants du pays, certaines tirées d'ouvrages traduits du français, comme celui de l'Histoire et description de la Nouvelle-France, du jésuite Charlevoix. Tout est mis en place pour accueillir le Canada dans le giron de l'Empire britannique, il ne reste qu’à prendre le territoire. Cette idée est largement répandue en Angleterre au début de la guerre, d'autant plus que le premier ministre fait de la guerre contre les colonies une priorité. Les journaux ne sont pas les seuls à préparer la population à la conquête du Canada; une œuvre frappe l'imagination, celle de Francis Hayman. Les peintures qui se retrouvent au Vauxhall créent un horizon d'attente sur ce que sera l'Empire, sur ses valeurs, mais aussi sur l'intelligence, la mansuétude et la magnanimité de ses généraux. Francis Hayman devient le chantre de Jonathan Tyers, mais surtout il se fait le relais d'un programme politique qui dépasse la simple composition esthétique. Voilà ce sur quoi il faut maintenant insister.

\section{The Surrender}

Que peut-on voir dans la représentation de Hayman, du moins, dans les deux modèles, bien différents l'un de l'autre, aujourd'hui conservés au Canada? Le premier, acquis par la Beaverbrook Art Gallery à Fredericton, est celui qui est le plus utilisé et le plus reproduit, sans doute parce que les descriptions que l'on nous donne du tableau original sont plus proches de ce premier modèle que du second, aujourd'hui la propriété du Musée canadien de la guerre à Ottawa.

La première illustration (illustration 1) est particulièrement sombre, la palette tourne autour de nuances de noir, de brun et de rouge. Au premier plan, le général Amherst, les bras ouverts, les yeux et le visage dirigés vers le bas, regarde un quatuor composé d'un vieillard et de trois enfants, dont un bambin. On remarque ici toute la volonté de l'artiste de faire du général un homme bon, compatissant. 
Alors que plusieurs militaires se plaisent à faire subir à la population les malheurs de la guerre, Amherst, d'un visage détendu, offre à ce groupe, dépourvu d'hommes en âge de faire la guerre, donc les victimes passives des combats, la grâce d'un geste apaisant.

Derrière ce groupe, se tiennent deux religieux, un chapelet à la main, signe de la tolérance que l'on se propose d'instaurer. Sous les pieds du général, un drapeau blanc flanqué de fleurs de lys, celui de l'ennemi vaincu. La France, dont les soldats sont absents, est ici réduite à un bout de tissu. Ainsi, ce n'est pas tant la France qui est vaincue qu'Amherst qui est vainqueur, vainqueur parce qu'il a su être celui qui portait les valeurs les plus nobles et les plus utiles. Le drapeau encercle une pierre carrée, en bas à droite, sur laquelle on peut lire difficilement : "Power exerted, Conquest obtained, Mercy shown, $M D C C L X$ ", sorte de message que l'on envoie au conquis et qui explique les raisons de la victoire. Cette victoire n'est d'ailleurs pas synonyme d'outrage, mais de clémence. À droite du tableau, se trouve un homme tenant un panier, dont on devine qu'il contient du pain. À l'extrême gauche, un soldat tient un drapeau qui, par sa présence et sa position dans le tableau, semble être une colonne sur laquelle repose la composition, sorte d'atlante placé de côté qui symbolise la protection offerte à la population nouvellement conquise.

Entre ce soldat et le général, au second plan, une foule, composée uniquement de femmes et d'enfants, dont tous les visages vont progressivement de la peur à l'imploration. Les personnages qui font face à Amherst ont toutefois une attitude presque calme. Pourtant, de ce groupe, une femme qui est tombée tient d'une main son enfant et, de l'autre, elle se soulève du sol; son regard est cependant toujours dirigé vers Amherst, sa seule planche de salut. Au troisième plan, à gauche du tableau, des individus le poing en l'air semblent réclamer quelque chose, mais deux soldats anglais, reconnaissables à leurs tricornes, les empêchent de venir troubler la scène principale. À droite, derrière le général, une tente, avec, d'un côté, trois arbres feuillus et, de l'autre, deux hommes, dont un soldat et ce qu'on devine être un Amérindien. La lumière, les attitudes et les gestes convergent 


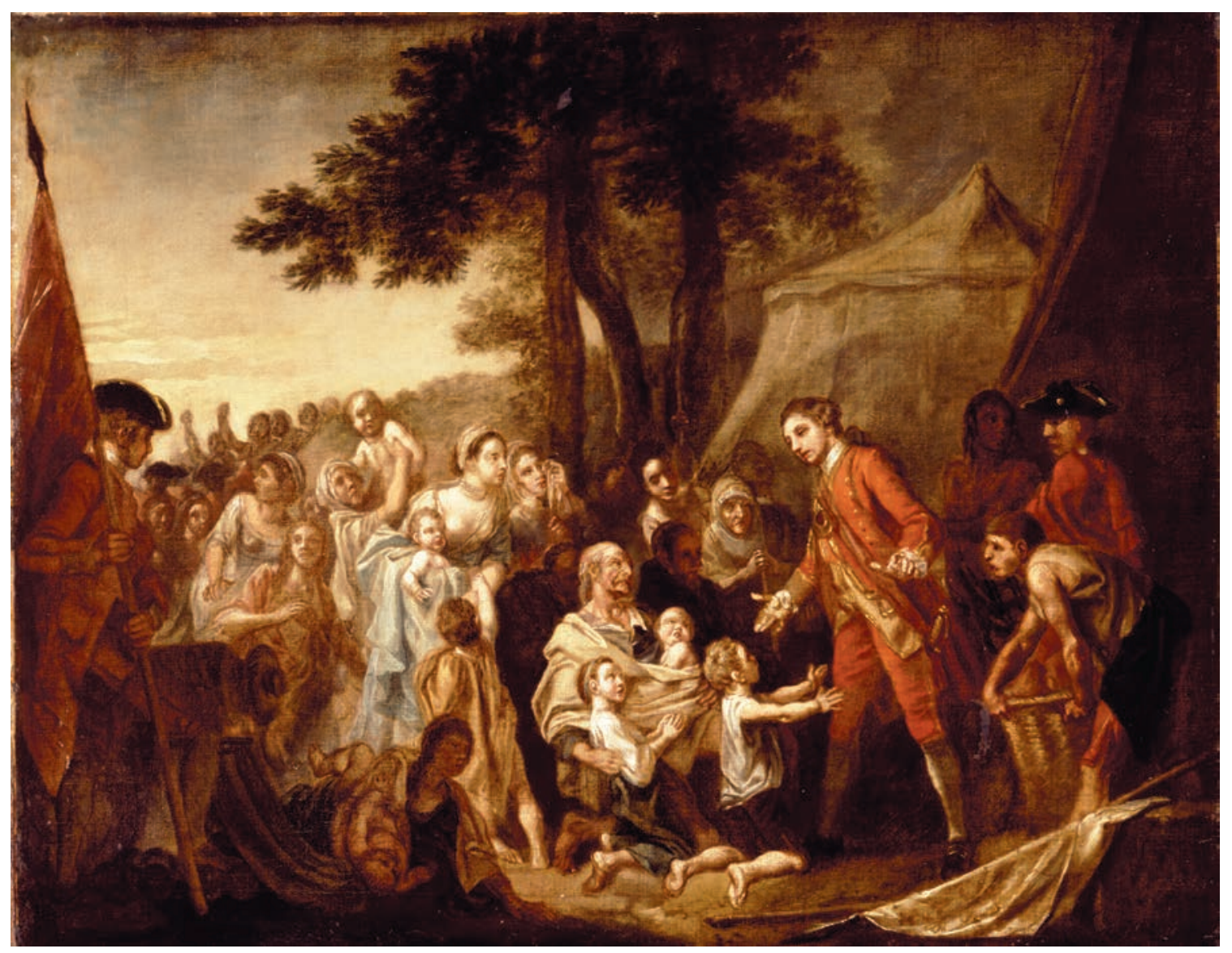

Ill. 1. Francis Hayman (1708-1776), The Humanity of General Amherst, huile sur toile, 90,4 cm x 111,5 cm, 1760 (Fredericton, La galerie d'art Beaverbrook/ La Fondation Beaverbrook (disputé, 2004)). 
vers un point central, la figure du général, qui résume par son seul geste toute l'attitude de l'Empire britannique à l'égard de la population : nous prendrons soin de vous.

La seconde représentation (illustration 2) présente des tons plus clairs : le bleu, le blanc et le vert tendre dominent. On retrouve toujours le général Amherst, cette fois la main gauche tendue vers une femme, la touchant presque, tandis que de la main droite il tient un papier sur lequel on peut lire : "Instructions to General Amherst". La clémence du général n'est donc pas le fait d'un seul homme, mais d'un royaume, voire d'une nation. L'homme à la corbeille d'osier y est toujours, cette fois à gauche, derrière lequel se retrouvent deux soldats discutant, dont l'un tient un drapeau. Derrière eux, on aperçoit une tente ainsi qu'un obélisque sur lequel est monté un jeune garçon, le visage apeuré, regardant derrière lui un homme qui lui tient la jambe et le pied. Le groupe de personnes venant se présenter à Amherst est plus important. Cette fois, tous les individus semblent implorer le général, les mains sont tendues, d'autres prient; d'ailleurs, les deux religieux sont toujours présents au centre du groupe.

Femmes et enfants sont en majorité. Les seuls hommes, à part les religieux, sont les enfants et le vieillard avec une canne à la main. Un autre homme est présent dans le coin inférieur droit, torse nu, la tête baissée. Il semble abattu, mais une femme s'empresse de le défendre en posant sur lui une main et en dirigeant l'autre vers le général. Serait-ce un soldat français qui, devant celui qui l'a vaincu, n'attend plus que la mort tandis que sa femme espère encore que celui-ci échappera à un sort qu'il n'a que trop mérité? La représentation semble abonder en ce sens. Au fond, un autre homme, tenant une croix, se confond avec le feuillage de l'arbre. Enfin, dans le dernier groupe, à l'extrême droite, des hommes, couteaux à la main, semblent poursuivre le groupe venu s'agenouiller devant Amherst. Des Amérindiens, des soldats français, des Canadiens? Difficile de distinguer des signes qui permettraient de confirmer une de ces hypothèses. Il semble cependant évident que certains refusent la défaite et entendent s'en prendre au vainqueur, mais les soldats anglais ont vite fait d'ériger 


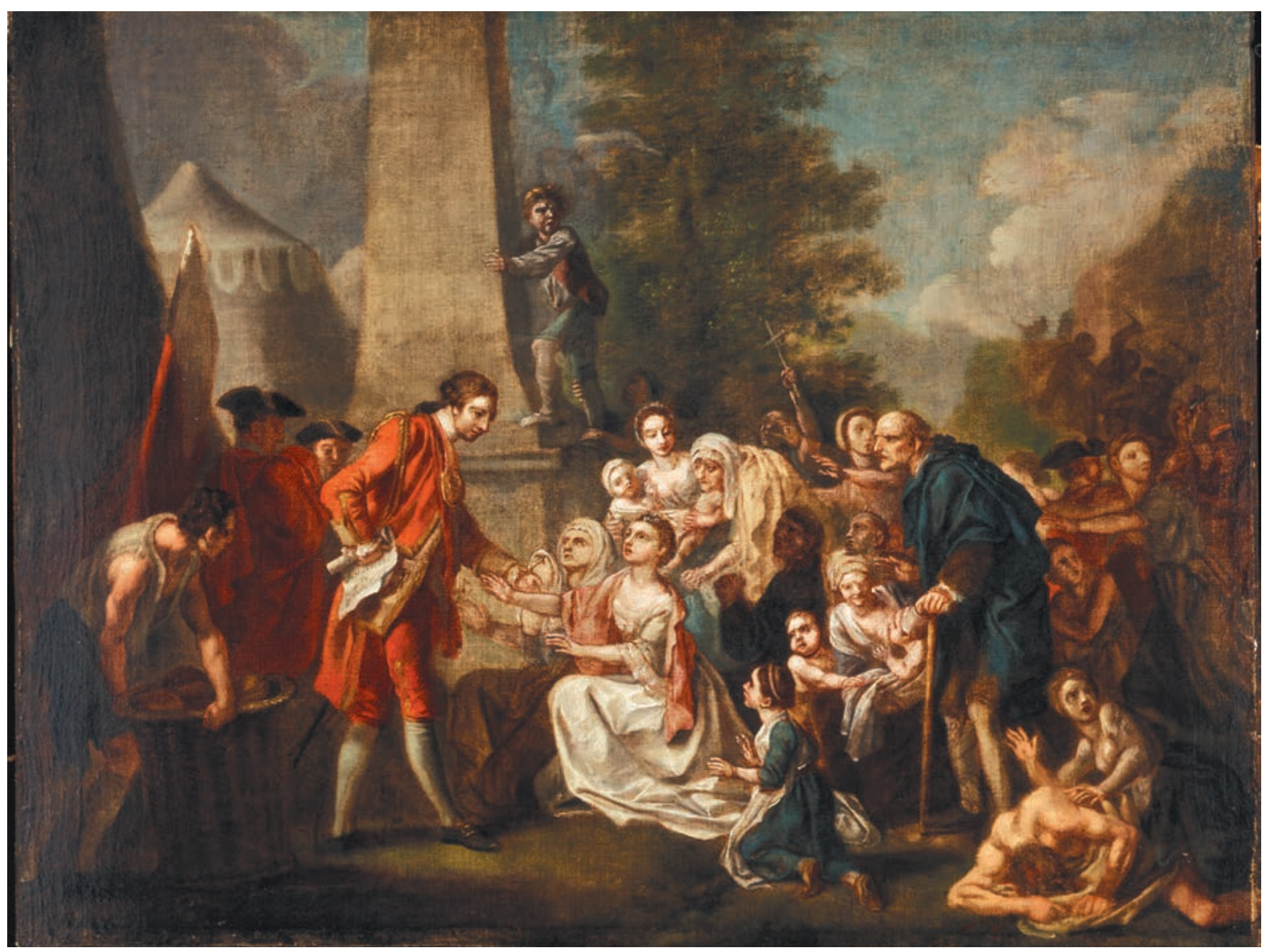

Ill. 2. Francis Hayman (1708-1776), The Charity of General Amherst, huile sur toile, $91,5 \mathrm{~cm} \times 71 \mathrm{~cm}, 1761$ (Ottawa, Musée canadien de la guerre, Collection d'art militaire Beaverbrook, n 19940037-001). 
une colonne d'hommes coupant l'accès au général, qui, de son côté, distribue ses bienfaits.

Une troisième représentation (illustration 3) existe de ce tableau. Sur un dessin de Robert Smirke, gravé par Philip Audinet et publié en 1792, l'œuvre porte ici le titre, reproduit en bas, de The Humanity of Gen. Amherst. Le fait qu'il existe une gravure de cette œuvre renforce l'idée qu'il y a eu une grande diffusion de cette image et qu'en plus d'être présente au Vauxhall, elle a fait l'objet d'une promotion dans l'espace privé. Que l'on ait gravé l'œuvre de Hayman montre également la grande portée que l'on a voulu donner à sa composition. Le titre de la gravure résume presque à lui seul tous les éléments évoqués dans les deux premiers modèles. Imprimée sur papier, la gravure fait 30,40 sur 20,30 cm (il est d'ailleurs possible d'en trouver une copie au musée Stewart à Montréal).

Associé à la Royal Academy, Smirke ${ }^{36}$ a réalisé, dans la seconde moitié du siècle, nombre de reproductions pour les ouvres de Shakespeare ou encore pour une édition des Mille et une Nuits. Le public visé par cette gravure ou encore la fonction de l'œuvre nous échappent. Elle est publiée par Harrison, éditeur à Londres qui se spécialise notamment dans l'édition de livres ornés de gravures. Il s'agit d'une libre adaptation de la peinture de Hayman, mais ses éléments principaux y sont reproduits. La pose du général est la même que celle de la deuxième étude. Cette pose est dorénavant doublée par celle d'un soldat retirant son chapeau et semblant acquiescer au désir d'Amherst. L'homme au panier d'osier laisse maintenant voir le pain que contient le récipient. À droite d'Amherst, on voit un Amérindien, mais ses traits semblent plutôt appartenir aux autochtones de l'Amérique du Sud. Si certains détails échappent au graveur, l'idée principale est toujours la même. À gauche du général, le groupe venu

\footnotetext{
${ }^{36}$ À propos de Robert Smirke, voir Cynthia E. Roman, « Robert Bowyer's Historic Gallery and the Feminization of the Nation ", dans Dana Arnold (dir.), Cultural Identities and the Aesthetics of Britishness, Manchester, Manchester University Press, 2004, p. 30-31.
} 


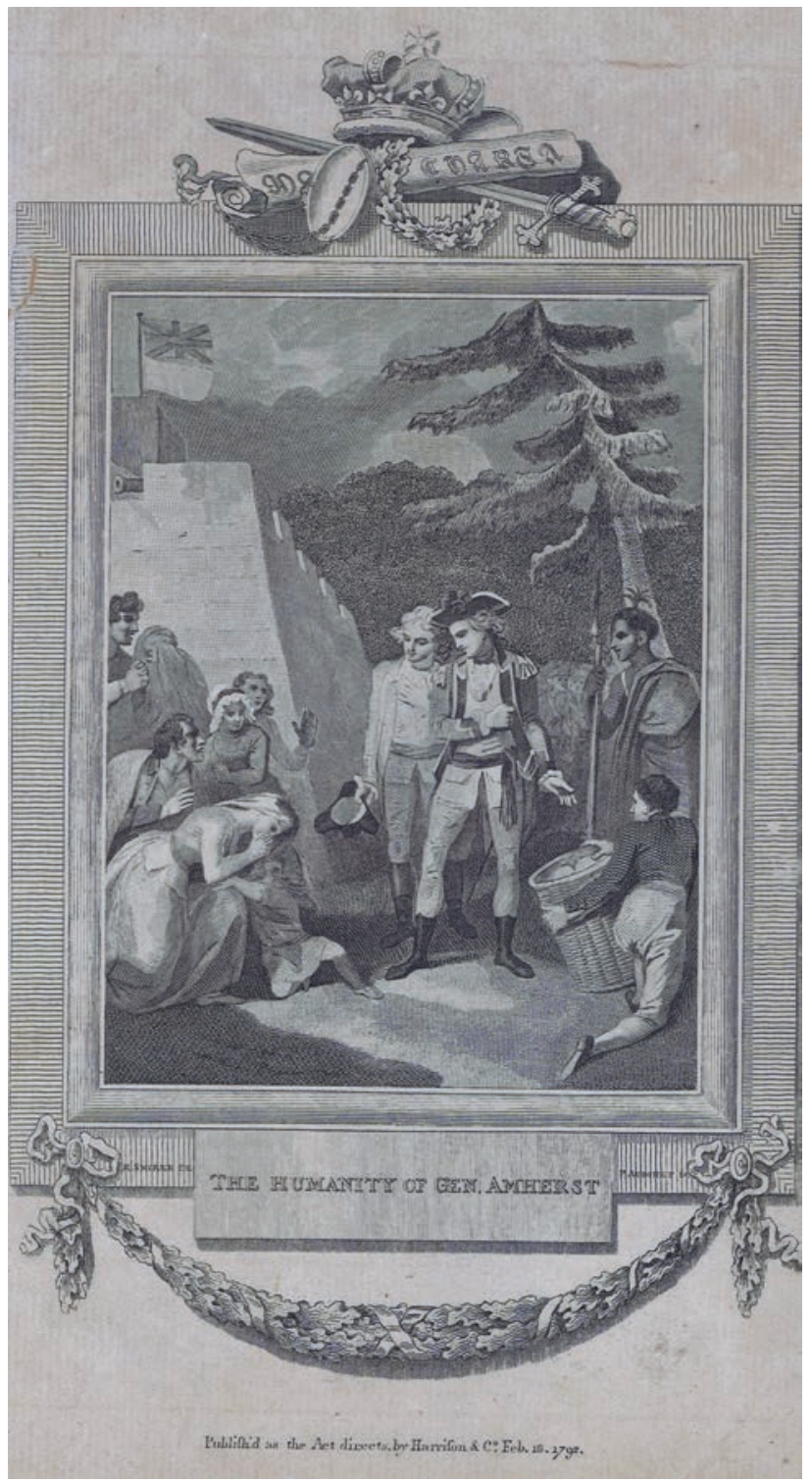

Ill. 3. Robert Smirke (1752-1845) (dessinateur) et Philip Audinet (1766-1837) (graveur), The Humanity of Gen. Amherst, d'après Francis Hayman, gravure, 30,40 cm x 20,30 cm, 1792 (Montréal, Musée Stewart). 
l'implorer est habillé, cette fois, selon la mode des années 1790-1800. Nouveauté singulière, la tente est maintenant remplacée par un fort, à gauche, au sommet duquel flotte un drapeau dont on voit distinctement l'Union Jack surmontant un drapeau blanc sans signe ou figure apparente. La France brille par son absence. Le fait de retrouver des gravures de l'œuvre et qui plus est, après la perte des colonies américaines (nouvellement les États-Unis d'Amérique), montre qu'il y a une véritable volonté d'ériger le général Amherst en vrai héros national. Pourtant, il faut, une fois encore, aller plus loin pour comprendre le contexte dans lequel s'insère l'œuvre et ce que celle-ci entend montrer.

\section{Une œuvre : quatre titres}

Deux modèles, une gravure, plusieurs éléments communs, d'autres qui diffèrent, mais la même idée, celle de la grandeur d'âme d'un général qui, plutôt que d'accabler son ennemi, préfere lui ouvrir les bras et faire preuve de mansuétude. Certes, le tableau montre relativement bien cette idée, mais elle est renforcée par le titre que l'artiste a donné à son œuvre. En introduction, j'évoquais les nombreux titres qui avaient été attribués à l'œuvre. Il convient de rétablir un peu les faits, du moins, ceux que nous connaissons. La description du Vauxhall de 1762 ne donne pas le titre exact de l'œuvre; on se contente de rappeler qu' " [i]t represents the surrender of Montreal in Canada ${ }^{37}$ ", sans spécifier davantage. Brian Allen, de son côté, affirme que le titre officiel est The Surrender of Montreal to General Amherst ${ }^{38}$. C'est d'ailleurs le titre donné par la majorité des historiens qui évoquent l'œuvre.

Les contemporains sont moins catégoriques. The Foreigner's Guide, guide de voyage édité en 1763, ne donne pas de titre exact, rappelant que cela représente " the Reduction of Canada, and General Amherst offering to the Canadians and French, Liberty and Security

\footnotetext{
${ }^{37}$ A Description of Vaux-hall Gardens, p. 24.

${ }^{38}$ Allen, Francis Hayman, p. 66.
} 
under the Government of Britain ${ }^{39}$ ". Georges-Louis Le Rouge, en 1770, dans les Curiosités de Londres et de l'Angleterre est tout aussi vague : «Il y a deux grands Tableaux de Hayman; l'un représente la conquête du Canada par le Général Amherst qui soulage les Canadiens $\&$ les François ${ }^{40}$. En revanche, le guide le plus connu, le plus utilisé et le plus réédité à Londres à l'époque, Ambulator, parle de "the surrender of Montreal, in Canada, to General Amherst ", titre qui, à partir de 1780, se généralise dans les guides de voyage. Les auteurs des principaux récits de voyage londoniens sont tout aussi vagues sur ce sujet. Kielmansegge, Grosley, Lacoste, Moritz et Chantreau restent tous muets sur le titre de l'œuvre, bien qu'ils parlent abondamment du tableau, notamment sur ce qu'il représente pour la nation britannique.

Le fait que l'œuvre disparaisse de la circulation avec la fermeture du Vauxhall en 1852 n'aide pas à démêler les choses. Ce qui semble évident, c'est qu'il s'est opéré une confusion entre les titres des deux modèles, dont le premier, celui de la Beaver, est souvent appelé Clemency of General Amberst, The Humanity of General Amberst ou encore The Surrender of Montreal to General Amherst, tandis que celui du Musée canadien de la guerre est désigné sous le titre de The Charity of General Amberst. Il ne convient pas de trancher cette question, les sources nous manquent pour le faire. En revanche, ce qu'il convient de retenir de ces différents titres est l'idée dont est chargée l'œuvre : celle d'humanité, de charité et de grandeur d'âme, dont j'ai parlé plus haut.

39 The Foreigner's Guide: Or, a necessary and instruction Companion both for Foreigner and Native, in their Tour through the Cities of London and Westminster = Le Guide des étrangers : ou le compagnon nécessaire \& instructif à l'étranger \& au naturel du pays, en faisant le tour des villes de Londres et de Westminster, $4^{\mathrm{e}}$ éd., Londres, Kent, Hope, Joliffe and Pote, 1763, p. 142.

${ }^{40}$ Georges-Louis Le Rouge, Curiosités de Londres et de l'Angleterre; par Le Rouge, ouvrage utile aux voyageurs. L'on y a ajouté un Abrégé de l'histoire \& des curiosités de la Hollande, Paris, Saugrain, 1770, p. 70. 
Les historiens vont reprendre cette idée, sans nécessairement exposer de façon systématique les interprétations qu'ils proposent. D'ailleurs, la majorité se sert de l'œuvre de Hayman pour illustrer tout bonnement la capitulation de Montréal, sans insister sur ce que l'artiste a voulu exprimer et sur le contexte de la peinture britannique. Pour Stephenson, "[t]he image of a magnanimous Amherst feeding hungry Canadians after the capitulation of Montreal contrasts with the commander's malevolent actions toward American Indian who resisted British control ${ }^{41}$ ". Laura Brandon va dans le même sens : "French soldiers are notably absent from the group of women, children, priests, the elderly weak. At the rear, an English soldier protects them from a group of naked "savages" bearing weapon. Behind Amherst, a servant brings in a large basket of bread which will be distributed "42 ", tandis que Carol Watts tâche de recontextualiser l'œuvre : "The surrender of Montreal to General Amherst portrayed a humanitarian act of relief, in which the General was shown extending his arms compassionately to the kneeling population of the city — both French and Indian — who had starved under siege ${ }^{43}$."

Ces interprétations, me semble-t-il, perdent de vue une idée essentielle. La chute de Montréal a lieu en septembre 1760 et le tableau est exposé dès le début de l'année suivante. Il est, bien sûr, impossible que l'artiste se soit rendu sur les lieux pour rendre compte des événements, d'ailleurs, les documents d'archives montrent qu'il était bien à Londres en 1760. Demeure alors une question fondamentale, celle de ses sources. De quoi s'est-il inspiré pour représenter la chute de Montréal ? Il convient d'analyser comment sont retranscrits les événements dans les journaux pour voir comment Hayman a pu s'en inspirer. Je considère les journaux, car il est plus que probable

\footnotetext{
${ }^{41}$ Stephenson, Clash of Empires, p. 85.

${ }^{42}$ Brandon, "Francis Hayman's The Charity of General Amberst ", p. 112. Voir également Allen, Francis Hayman, p. 66.

${ }^{43}$ Carol Watts, The Cultural Work of Empire: The Seven Years' War and the Imagining of the Shandean State, Edinburgh, Edinburgh University Press, 2007, p. 65.
} 
que l'artiste les ait consultés, lui qui a travaillé activement pour certains d'entre eux, dont les rééditions du Tatler.

\section{Les périodiques londoniens et la chute de Montréal}

Le nombre de périodiques britanniques va sensiblement augmenter au cours de ces années. Des publications comme le London Magazine, le Gentleman's Magazine ou encore le British Magazine orientent et définissent de plus en plus l'opinion publique en rapportant les nouvelles locales, nationales et internationales. Les guerres d'outreAtlantique deviennent peu à peu des feuilletons que l'on suit de semaine en semaine. Plusieurs de ces journaux ont fait ici l'objet d'analyse. J'ai dépouillé systématiquement la presse londonienne en rapport aux événements de la guerre au Canada et, plus spécifiquement, les articles relatant la capitulation de Montréal, afin de comprendre ce que Hayman a pu représenter d'après les informations disponibles à Londres en 1760 et 1761 .

Les journaux londoniens commencent à parler de Montréal bien avant la capitulation. Dans The British Magazine, on vante, à de nombreuses reprises, les avantages de cette terre, en rappelant l'histoire et l'avancée des troupes de Sa Majesté. On peut ainsi lire que " [t]he town of Montreal, which stands in a Island of the river, is ill fortified; may be attacked by land and water at the same time, and cut off from all communication with the country, so as to be starved in a few days ${ }^{44}$ ". La victoire semble une évidence, la puissance de l'armée et l'intelligence des officiers et généraux, déjà démontrées lors de la prise de Québec, laissent entrevoir que Montréal sera une proie facile. D'ailleurs, dès 1759, le Universal Magazine présente une carte de cette ville (illustration 4). Le Gentleman's Magazine publiera, après la victoire, en octobre 1760 une carte «showing Gen. Amherst's march to Montreal, and a plan of the town" (illustration 5). Il existe donc en Angleterre

\footnotetext{
44 "Reflections on the Present Posture of our Affairs in North America ", The British Magazine of Monthly Repository for Gentlemen \& Ladies for January 1760, London, Rivington, Fletcher and Payne, 1760, p. 84.
} 


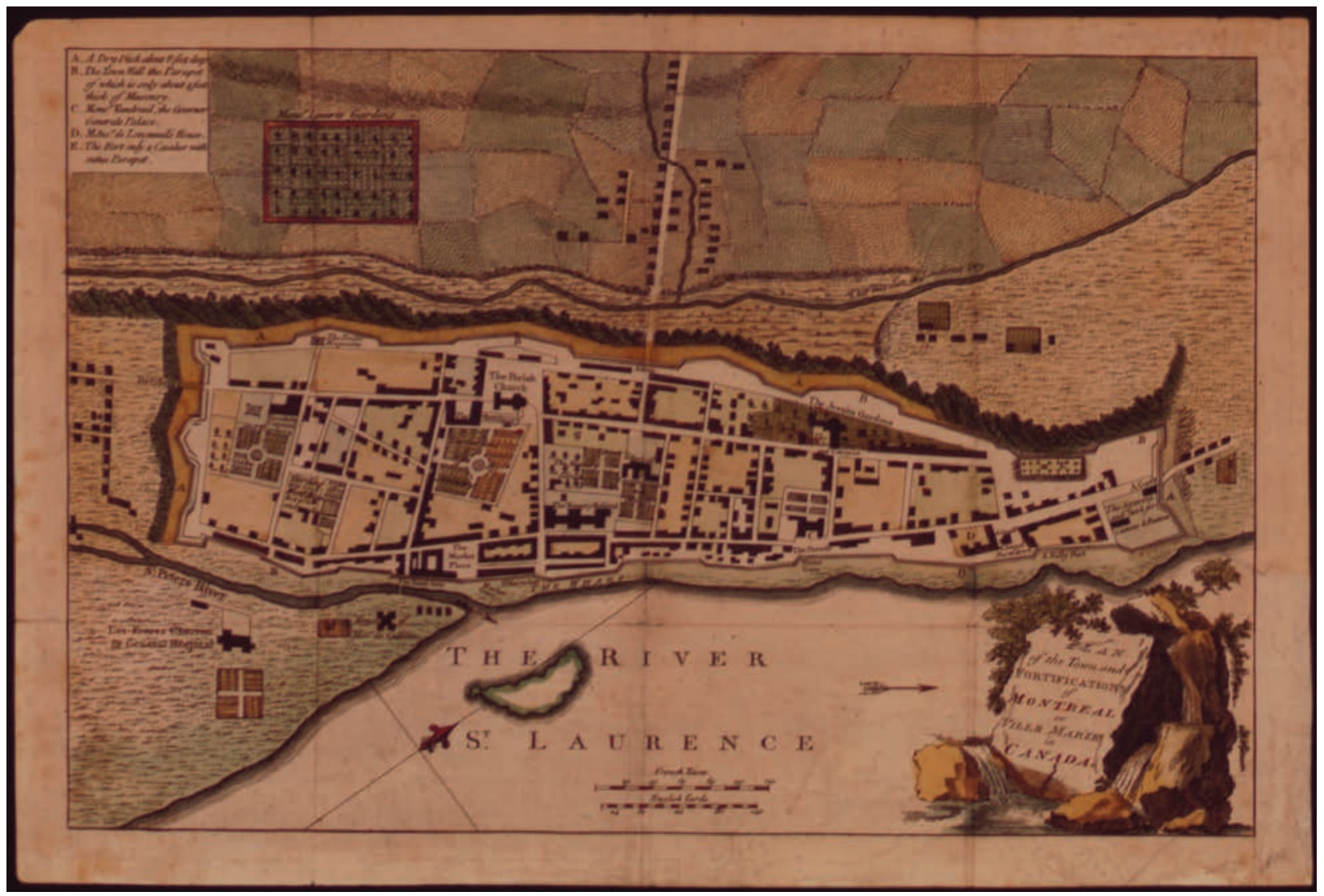

Ill. 4. "Plan of the Town and Fortifications of Montreal or Ville Marie in Canada ", Universal Magazine, novembre 1759 (Bibliothèque et Archives Canada, RBC Financial Group Collection, n 0027649). 


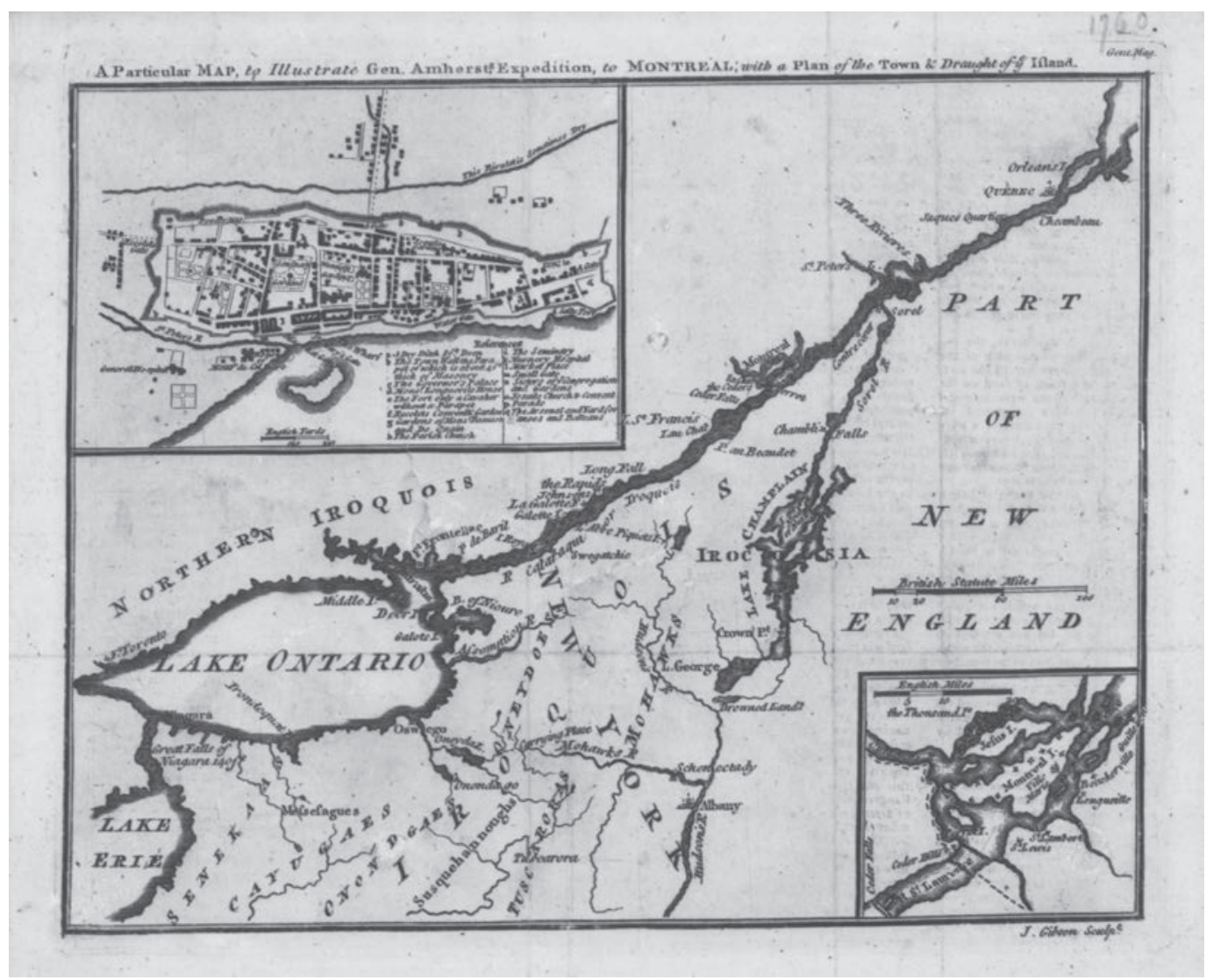

Ill. 5. «A Particular Map, to Illustrate Gen. Amherst's Expedition, to Montreal, with a Plan of the Town \& Draught of ye Island ", Gentleman's Magazine, octobre 1760 (Bibliothèque et Archives Canada, Alexander E. MacDonald Canadiana Collection, NMC 6656). 
des outils pour reconnaître le territoire canadien, mais aussi la disposition de la ville de Montréal.

Le London Magazine est un des premiers journaux qui, dès octobre 1760, rapporte les événements entourant la capitulation montréalaise. Le style, mais aussi le type d'informations permettent de comprendre un peu mieux le thème qu'a choisi d'exploiter Francis Hayman, mais également les valeurs qu'il a voulu célébrer à travers la représentation d'Amherst. Si le 8 septembre 1760 l'armée britannique peut entrer dans Montréal, ce n'est qu'en octobre 1760 que le public londonien est mis au courant des différentes péripéties entourant cet événement. De janvier à septembre, les articles permettant de croire que la chute de Montréal est proche sont nombreux. Le numéro d'octobre est d'ailleurs presque entièrement consacré à Montréal. Pour se représenter un peu mieux la ville, on publie « $a$ large and accurate Plan of the Town and Fortifications of Montreal in Canada, and an elegant View of the Same Town ", avec une petite vignette d'une vue panoramique.

La gloire britannique est encore évoquée dans deux textes du même numéro du London Magazine. Le premier est une "[d]escription of the City of Montreal, and its Environ". Si on y apprend que " the fortifications are pretty strong, being surrounded by a wall, flanked with eleven redoubts", on ajoute que "the French have never been able to establish any stable commodity to answer their demands on their mother country ". En revanche, les liens unissant les colonies britanniques à la ville sont de plus en plus forts et montrent à quel point cette conquête est logique et procède de l'idée selon laquelle l'Empire britannique est basé sur le commerce et l'échange : "The French have found by experience, that it was cheaper for them to purchase their goods of the New York merchants, than to have them from their own country. " Ce commerce ne pourra que prospérer, car « Canada will be ceded to us; and consequently, that we shall have no European enemy to fear on 


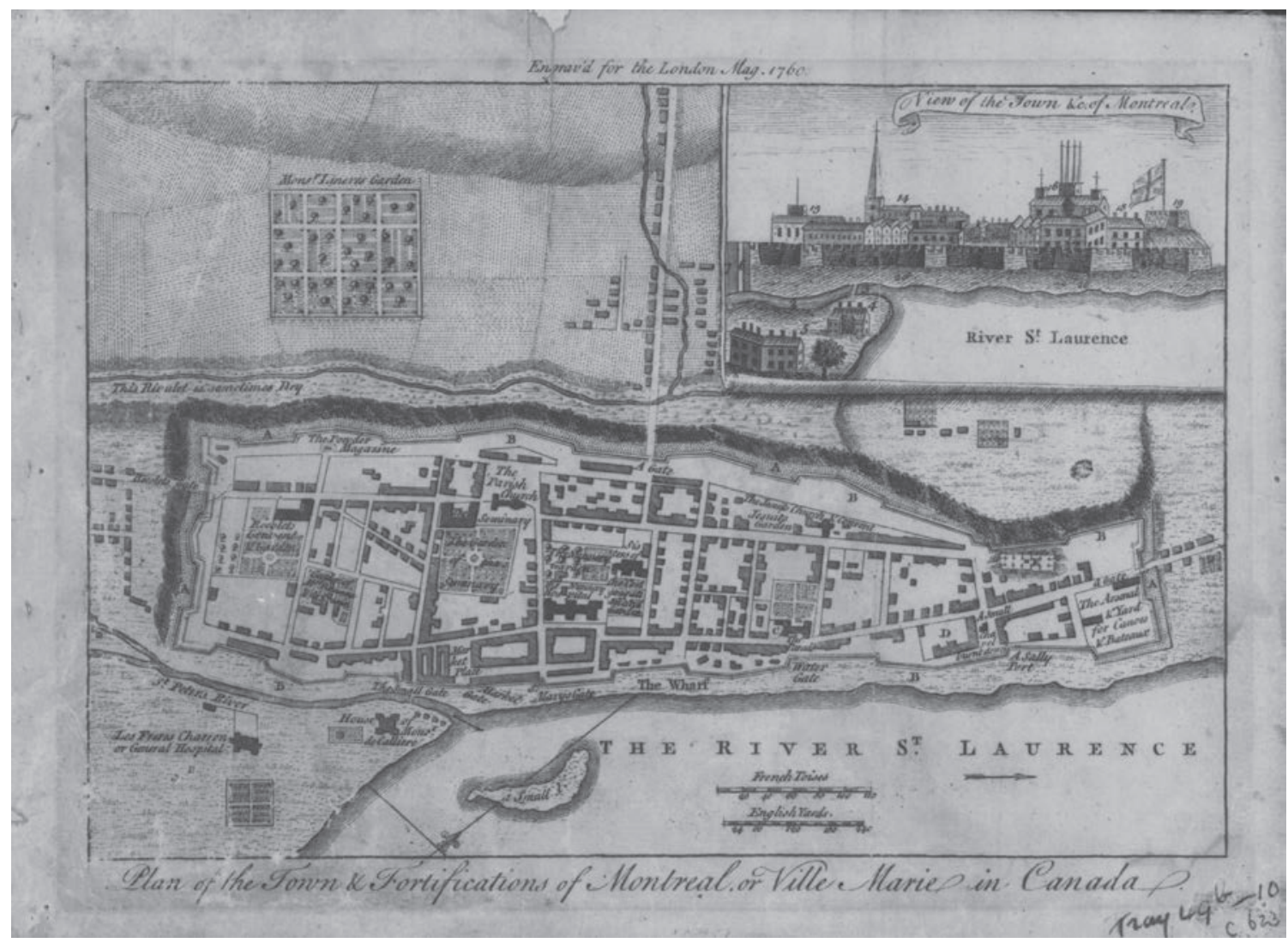

Ill. 6. «Plan of the Town \& Fortifications of Montreal or Ville Marie in Canada ", The London Magazine: Or, Gentleman's Monthly Intelligencer, octobre 1760 (Bibliothèque et Archives Canada, Collection nationale de cartes et plans, NMC 133355). 
that vast continent ${ }^{45}$ ". L'auteur, anonyme, se rattache ici à tout un courant philosophique en Angleterre, voulant que le commerce fonde l'Empire, lui donne son identité et lui permette d'assurer la réussite matérielle.

Le second texte du London Magazine est plus polémique et prend le parti de montrer en quoi les valeurs britanniques, plus que les armes, ont su triompher de l'ennemi français en territoire nordaméricain. Le 16 octobre, lord Mayor reçoit William Pitt pour le féliciter de cette conquête. Sir William Moreton prononce un discours dont un passage est ici retranscrit. On peut lire :

The conquest of Canada, so heroically begun at Quebec [...] and so happily completed without the effusion of human blood at Montreal; at the same time that it reflects the highest honour upon the wisdom and vigour of your majesty's councils, upon the conduct, fortitude, and activity of your commanders, and upon the bravery and discipline of your fleets and armies, is an event of the utmost importance to the trade and manufactures of these kingdoms [...].

À la grandeur britannique, il oppose la barbarie française : " [...] against the continual encroachments and unparalleled barbarities of a restless and insidious enemy, even more dangerous in peace than war. " On souligne encore à quel point les chefs militaires ont agi honorablement face à un ennemi qui n'aurait mérité qu'une mort violente. Des valeurs d'humanité sont opposées : " [...] towards enabling your Majesty to preserve this valuable acquisition, and effectually to prosecute the various and extensive services of this just and necessary war, and to dictate to the aggressors the terms of a safe and honourable peace ${ }^{46}$." Plutôt que la barbarie française, c'est ce deuxième aspect (l'humanité) que Hayman choisit de représenter. Le corps, l'expression et le tendre sourire d'Amherst confirment cette

45 "A Description of the City of Montreal and its Environ ", The London Magazine: Or, Gentleman's Monthly Intelligencer for October 1760, London, R. Baldwin, 1760, p. 543-545.

46 The London Magazine: Or, Gentleman's Monthly Intelligencer for October 1760, p. 549. 
volonté de montrer une noblesse d'âme typiquement britannique. La population opprimée, représentée par des hommes et des femmes, ne peut que s'incliner devant un tel déploiement de grâce et de mansuétude.

À cette tendance qui fait de l'expansion coloniale un facteur déterminant dans le développement du commerce et à celle vantant les valeurs britanniques de paix et de courage, il faut ajouter l'importance que prend la figure du général Amherst en Angleterre. Certes, après le 8 septembre 1760, il est reconnu comme celui qui a réussi à faire capituler Montréal et le Canada. Ses réussites sur le territoire nord-américain font de lui une figure emblématique des réussites militaires. Toutefois, cela ne date pas de 1760, car en 1759, Horace Walpole écrit :

Galfridus Amhersta Ticonderagicus, we should be quoted a thousand years Hence as the patterns of valour, virtue, and disinterestedness; for posterity Always ascribes all manner of modesty and self-denial to those that take The mostpainstoperpetuate theirownglory...Inshort, wewant but a little More insolence and a worse cause to make us a very classic nation ${ }^{47}$.

Notons au passage les références à l'Antiquité, qui sont intrinsèquement liées aux valeurs qui définissent l'Empire britannique. Puis, en décembre 1760, paraît, dans le London Magazine, "A Martial Song: A Song on the Taking of Montreal » (illustration 7), sur des paroles de John Lockman, celui-là même qui régale le Vauxhall de Jonathan Tyers de chansons en tout genre.

Amherst est élevé au rang de héros : "Amherst's praise, / And spread round the globe Amherst's praise. I [...] His progress he takes, I With Montreal full in his eyes, / The French would, in vain, / Or Indians, restrain / His troops, who to victory fly. / [...] Great blow to the commerce

47 " Horace Walpole to Strafford, September 13, 1759 », The Yale Editions of Horace Walpole's Correspondence, édité par W. S. Lewis, Yale, Yale University Press, 1971, vol. 25, p. 294. 
of France! / [...] The French are undone; / And now Canada's won, I Britannia shall there fix her throne ${ }^{48}$. " Les sources vives de l'œuvre de Hayman apparaissent ici clairement, ce sont celles d'un homme, Amherst, ayant su être généreux envers une population délaissée par la mère patrie qui, dès les premiers mouvements d'ouverture de la part du général, se met à ses pieds pour chanter sa gloire. Qu’a gardé Hayman des représentations et des descriptions du territoire montréalais? Rien, aucun des éléments évoqués dans les journaux n’apparaissent dans l'œuvre. En fait, la réalité du territoire importe peu; pour celui qui regarde l'œuvre au Vauxhall, seules comptent la grandeur et la clémence du général. De plus, Hayman entend faire le pont avec des figures classiques rappelant les hauts faits d'armes d'un autre empire, romain celui-là. Montréal n'est plus qu'un paravent à une idée qui dépasse et englobe tout.

\section{Des regards contemporains sur l'œuvre}

Entre ce que veut dire un artiste et la manière dont il est compris, il y a souvent un écart considérable. Je me propose ici de voir comment les contemporains ont apprécié et interprété The Surrender of Montreal de Francis Hayman. Les premiers documents qui donnent un sens à l'œuvre sont les guides de voyage londoniens avec, dans un premier temps, A Description of Vaux-hall Gardens de 1762. La position du corps d'Amherst est claire, c'est celle d'un homme qui accueille généreusement une population meurtrie par la guerre. En plus de la scène représentée, le tableau évoque une certaine idée des victoires anglaises, mais surtout les valeurs propres à la nation britannique : "[...] but general Amherst who agreeable to his instructions and his own humane disposition, grants the whole of their suit, commands them to be replaced in their possessions, orders bread to

\footnotetext{
48 "A Martial Song: A Song on the Taking of Montreal, song by Mr. Lowe; set by Mr. Worgan; the Words by Mr. Lockman. Presented to his Royal Highness the Prince of Wales ", The London Magazine, décembre 1760, p. 660.
} 

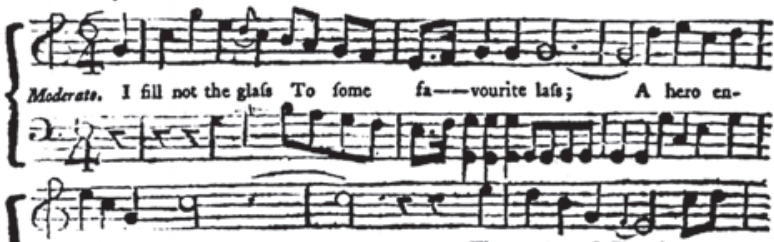
groffes my bys: Thy trumpet, O Fame! His

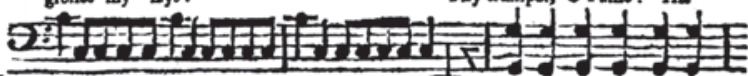
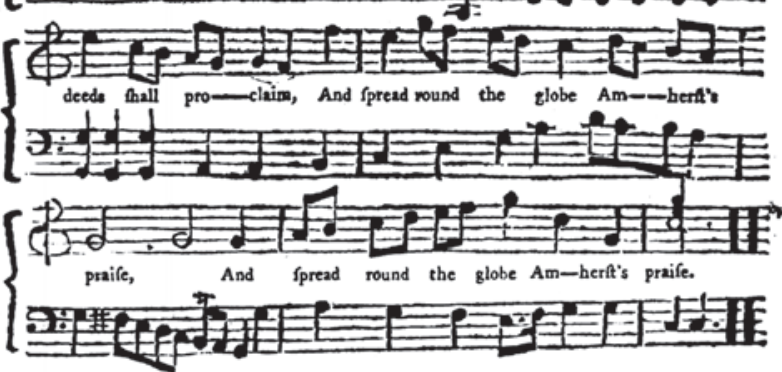

Through woods, and o'er lakes, His progref's he takes,

With Montreal full in his eyea

The French would, in vain,

Or Indians, reftrain

His troops, who to vietory fly.

Cape-Breton our own, 3 .

Gallia's fith'ry's o'crthrown,

Chief nurfery of her marine :

Invation, that joke,

Will thence end in imoke, And Britain fill teign ocean's queen.

The Indians and we

Shall henceforth agree;

Thus our manutactures advance ,

Our fues, to their cott,

See their rich fur -trace loft, -

Great blow to the commerce of France!
Triumphant, with pride,

O'er ocean we ride;

Not a fingle attempt now mifcarries s

To our ravifh'd eyes

Creffy, Agincourt, rife,

And the days of our Edwards and Harrge'

JuR George! O for thee,

The Fates did decree,

A reign will eternally thine :

The fam'd conquefts told

In our annals of old, Are already eyuall'd in thine.

O'erwhelm'd with fad iear,

Sce Gallia, in tears,

The lof's of Munireal bemoan:

The French are undonc;

And now Canada's vion,

Bitannia fhall there fix her throne,

But hark! heav'n-bnrn peace

Bid's war's hor rors ceafe;

And to where the rodiefs defecads?

Het tharms all aciore;

Hinvat biond v̂resons no more;

And foes, lor.g ccesectirg, are fricade.

Ill. 7. " A Martial Song: A Song on the Taking of Montreal, song by Mr. Lowe; set by Mr. Worgan; the Words by Mr. Lockman. Presented to his Royal Highness the Prince of Wales ", The London Magazine, décembre 1760, p. 660. 
be destributed amongst them and by a series of other benefits convinces them that clemency is the genius of the British nation ${ }^{49}$. "

Les autres guides de voyage sont tout aussi explicites. La majorité des auteurs se contente de reprendre grossièrement les termes de cette première lecture en l'adaptant quelque peu. The Foreigner's Guide, qui offre des informations en anglais et en français (car nombre de voyageurs français traversent la Manche pour visiter Londres), est plus détaillé dans la langue de Shakespeare que dans celle de Molière. On retrouve ainsi en français la description suivante : «le Premier représente le Conquest de Canada par le General Amherst, qui soutient les pauvres Canadians \& François, et leur donne des Viands, au même tems il leur promet la Protection et la Liberté de la Grande-Bretagne $[s i c]^{50}$ ", tandis que dans une note en bas de page, plus explicite et uniquement en anglais, on lit :

General Amherst [is] surrounded with a Multitude of miserable French and Canadians, who having abandoned their Dwellings, throw themselves at his Feet, imploring Compassion, Protection and Relief. The General, empower'd by his Instruction, and inclin'd by his natural Disposition, grants the whole of their Suit, and more; commanding them to be replaced in their Possessions, and supply'd with Provisions, and convincing them by a Series of Benefits, that Clemency is the Genius of the British Nation, and Liberty and Security the natural Fruits of the British Government ${ }^{51}$.

On va plus loin, en exaltant la fierté et la puissance. C'est aux Britanniques que cette œuvre s'adresse et c'est pour eux qu'elle a été faite; il faut donc chanter les gloires sans modestie. Le Français Georges-Louis Le Rouge, dans Curiosités de Londres et de l'Angleterre ${ }^{52}$, reprendra la première partie de la description qui se trouve dans The Foreigner's Guide, en omettant toutefois la seconde, tout comme le Ambulator, très utilisé par les étrangers de passage à Londres, qui,

\footnotetext{
${ }^{49}$ A Description of Vaux-hall Gardens, p. 25.

${ }^{50}$ The Foreigner's Guide, p. 143.

${ }^{51}$ Ibid., p. 142.

${ }^{52}$ Le Rouge, Curiosités de Londres, p. 70.
} 
après la Révolution américaine, ne fera que signaler la victoire en terre canadienne: "The first represents the surrender of Montreal, in Canada, to General Amherst. On a stone, at one corner of the picture, is this inscription Power exerted, Conquest obtained, Mercy shown! $1760^{53}$. "Liberté, sécurité, bénéfices, protection, compassion sont quelques-unes des idées qui définissent l'Empire et qui se retrouvent dans les descriptions de l'œuvre. Celui qui sait lire l'œuvre et plus encore la langue de Shakespeare en est ici doublement convaincu.

Les auteurs des récits de voyage sont, eux aussi, très loquaces à propos du tableau de Hayman. Trois Français (Grosley, Lacoste et Chantreau) et un Allemand (Moritz), commentant l'œuvre, en profiteront pour disserter sur le pouvoir, les Anglais, leurs conquêtes et la manière de les représenter. Les voyageurs français ne sont pas nécessairement choqués de la représentation : "Une tendre \& noble compassion se peignent dans la physionomie $\&$ dans toute l'attitude de ce général », rapporte le Champenois Grosley, avant de s'interroger : " [... ] j'y trouvois souvent des François arrêtés par de charitables Anglois, qui leur en donnoient l'explication avec tous ses détails \& tous ses agrémens. J'en entendis un demander à un garçon sculpteur, ce que signifioient les trois fleurs-de-lys jettées sur le drapeau qui servoit de tapis de pied au Général. » Plutôt que de se plaindre, Pierre-Jean Grosley réfléchit ici à la fonction de représentation du pouvoir royal avec, comme modèle, un des rois qui a su s'exposer et se faire représenter le mieux aux yeux des siens et des étrangers :

Louis XIV est le premier des souverains modernes qui, par des monumens durables, ait ainsi insulté aux vaincus : ils le lui ont bien rendu depuis. A la vue des monumens Anglois, nous pouvons dire avec les Grecs vainqueurs des Troyens \& gémissant de leurs victoires :

Fastûs pœnas expendimus omnes. Aneeid, Lib. IX.

${ }^{53}$ Ambulator: or, a Pocket Companion in a Tour Round London, London, Scatcherd, 1800 , p. 218. 
L'animosité, entretenue \& perpétuée par ces monumens, saisit toutes les occasions pour se montrer \& se faire sentir : elle iroit jusqu'au mépris chez des gens qui, absolument nouveaux dans les affaires du monde, ignoreroient combien sont inconstantes les faveurs de la fortune ${ }^{54}$.

Jean-Aimé de Lacoste, de son côté, trouve l'œuvre médiocre. Un homme avec qui il s'entretient lui déclare : "Les Anglois, me dit-il, n'ont pas moins d'actions éclatantes à mettre dans leurs annales que les autres peuples de l'Europe; mais, en vérité, ils multiplient tellement les trophées, que l'étranger répugne à leur rendre hommage. "Puis, l'auteur ajoute cette réflexion :

Je ne suis pas cet étranger, lui répondis-je, en souriant; la modestie n'est pas moins louable dans un corps que dans les individus, cela est incontestable; mais je crois que sous le régime républicain, la jactance du gouvernement a principalement pour but d'exciter cette exhaltation de l'esprit qui fait les héros; et, en faveur de ce motif, je pardonne à l'Angleterre ce grand nombre de monuments d'orgueil, quoique érigés, pour la plupart, aux dépens de l'amour-propre François ${ }^{55} \ldots$

Pierre Nicolas Chantreau, en 1792, soit après la chute de la monarchie française, va même jusqu'à souhaiter ce type d'art patriotique pour la France : "Quand le pinceau de nos artistes, las de flatter les grands ou les mannequins qui suppléent par leur or aux grands noms, s'employera à nous tracer les véritables grands hommes et leurs actions, nous verrons comme les Anglois, nos salles d'assemblées décorées de sujets nationaux, propres à faire naître cet esprit public dont nous avons tant de besoin, pour être libres, ou dignes de l'être ${ }^{56}$. " Les voyageurs français reconnaissent aisément le message, mais ce qui importe le plus, c'est la fonction de l'œuvre, qui est un

${ }^{54}$ Pierre-Jean Grosley, Londres, nouvelle édition, revue, corrigée \& considérablement augmentée, tome premier, Lausanne, 1774, p. 190.

${ }^{55}$ Jean-Aimé de Lacoste, Voyage philosophique d'Angleterre, fait en 1783 et 1784, tome premier, Londres, 1786, p. 153.

${ }^{56}$ Pierre Nicolas Chantreau, Voyage dans les trois royaumes d'Angleterre, d'Écosse et d'Irlande, fait en 1788 et 1789, Paris, Briand, 1792, t. II, p. 129. 
livre ouvert sur l'ambition anglaise, bien légitime, car elle est la seule à pouvoir y prétendre, comme on l'écrit si clairement. Le tableau de Hayman et les guides de voyage parlent ici la même langue, celle de l'exaltation nationale.

Tous semblent reconnaître la fonction nationale de ces œuvres et l'utilité d'un message bien construit qui montre une Angleterre toute-puissante. Seule l'interprétation de Charles P. Moritz differe légèrement, évoquant même l'émotion dont le spectateur est envahi à la vue du tableau :

If you look at this painting with attention, for any length of time, it affects you so much, that you even shed tears. The expression of the greatest distress, even bordering on despair, on the part of the besieged, the fearful expectation of the uncertain issue, and what the victor will determine concerning those unfortunate people, may all be read so plainly, and so naturally in the countenances of the inhabitants who are imploring for mercy, from the hoary head to the suckling whom his mother holds up, that you quite forget yourself, and in the end scarcely believe it to be a painting before you ${ }^{57}$.

L'œuvre de Hayman remplit la première fonction pour laquelle elle a été commandée, c'est-à-dire provoquer un sentiment de fierté et souligner les bienfaits des conquêtes britanniques. Jonathan Tyers, quand il a commandé l'œuvre, et ce, même avant la fin de la guerre, sert cette grande idée qui avait cours au milieu du XVIII ${ }^{\mathrm{e}}$ siècle et remarquablement bien résumée par Kathleen Wilson : "Imperial struggles were viewed at home as battles over the national character $[\ldots]^{58}$. " On expose un programme pictural et textuel conçu pour influencer le Londonien du midi du siècle.

${ }^{57}$ Charles P. Moritz, Travels, Chiefly on Foot, through Several Parts of England, in 1782, Described in Letters to a Friend by Charles P. Moritz a Literary Gentleman of Berlin, translated from the German by a Lady, $2^{\mathrm{e}}$ éd., Londres, Robinson, 1797, p. 37.

${ }^{58}$ Wilson, The Sense of the People, p. 202. 


\section{"Clemency is the Genius of the British Nation"}

The Surrender of Montreal présente aussi un autre niveau de référence dans la construction de l'identité impériale : l'Antiquité. Le retour à l'Antiquité n'est pas propre à la Grande-Bretagne; il s'agit plutôt d'une caractéristique des Lumières. Artistes français, italiens, allemands et anglais vont chercher dans la Rome impériale ou encore dans la Grèce d'Alexandre des thèmes qui servent à exprimer les passions humaines, mais aussi, et c'est l'apport du XvIII ${ }^{\mathrm{e}}$ siècle, l'esprit patriotique. En 1733 est fondée dans l'Angleterre des Hanovre la Society of Dilettanti, qui accueille les gentilshommes de passage en Italie, mais qui finance aussi des fouilles et des publications portant sur les ruines et les édifices de l'Antiquité. Ajoutons à cela les nombreuses publications de collectionneurs qui expliquent, recensent et donnent de la valeur aux œuvres d'art antiques. De 1760 à 1763, James Macpherson écrit et publie des poèmes épiques qu'il attribue à Ossian, un barde imaginaire qui aurait vécu au III ${ }^{\mathrm{e}}$ siècle. Il plonge alors l'Europe dans une crise " ossianite » aiguë.

Cette influence de l'Antiquité va profondément marquer Francis Hayman. Les spécialistes s'accordent pour reconnaître une filiation entre The Surrender of Montreal et La famille de Darius aux pieds d'Alexandre le Grand (voir l'illustration 8) ${ }^{59}:$ " [...] there is a direct compositional link to French artist Charles LeBrun's The family of Darius before Alexander of 1660-1, in Versailles, where Alexander behaves charitably towards the defeated Persians ${ }^{60}$ ", écrit Laura Brandon. Jonathan Conlin renchérit : " "The Continence of Scipio" and "Alexander in Darius' Tent" were incidents from ancient history which proved popular with seventeenth-and early eighteenth-century painters such as Nicolas Poussin, Charles Le Brun and Sebastiano Ricci. Like the aforementioned classical texts, they provided examples of model behaviour for royal patrons eager to style their military triumphs in the manner of their great Roman

\footnotetext{
${ }^{59}$ Voir Jean Vittet et al., La tenture de l'histoire d'Alexandre le Grand, Paris, Éditions de la RMN, 2008.

${ }^{60}$ Brandon, "Francis Hayman's The Charity of General Amherst ", p. 112.
} 


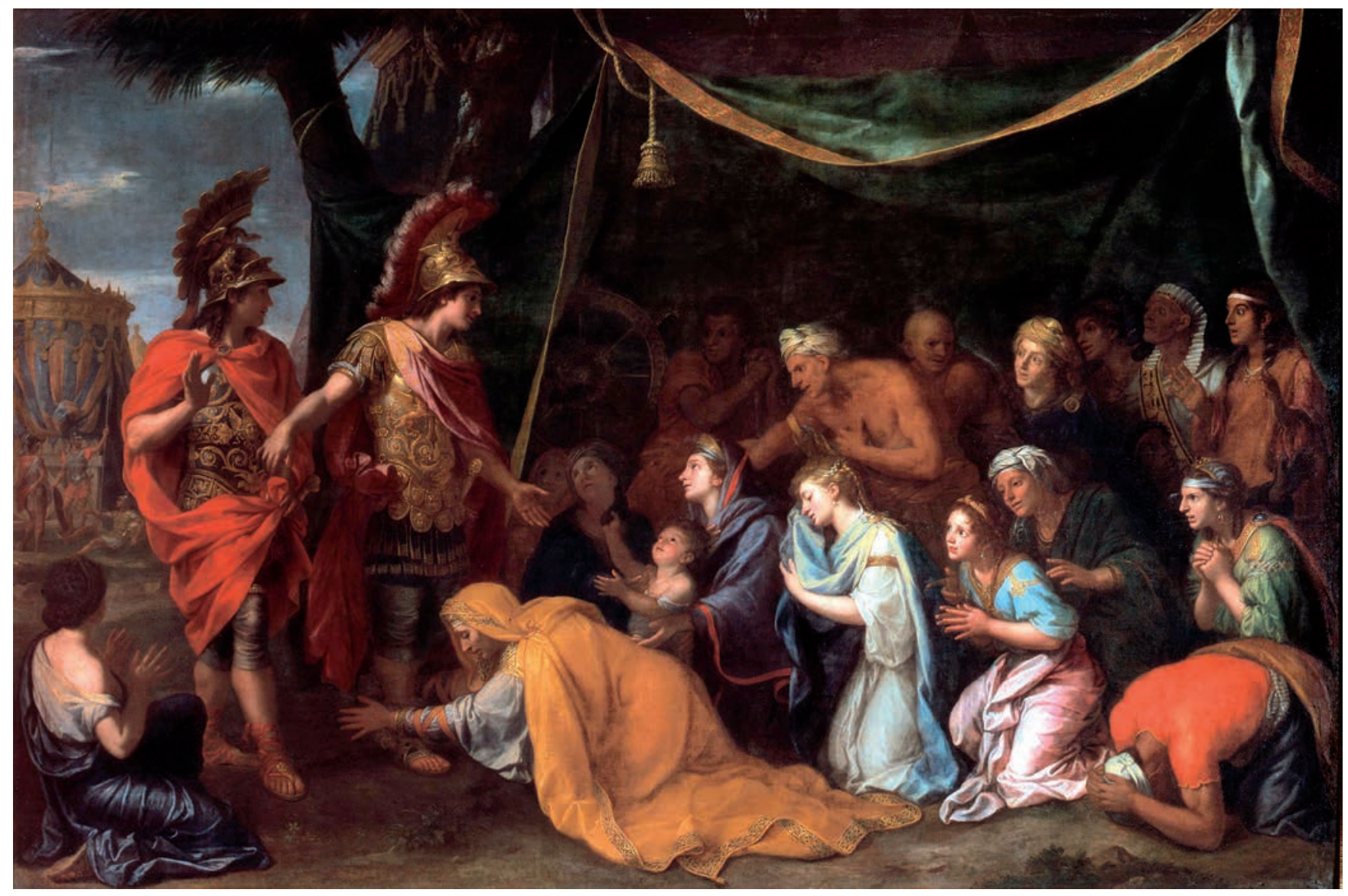

Ill. 8. Charles Le Brun (1619-1690), La famille de Darius aux pieds d'Alexandre dit " la tente de Darius ", huile sur toile, 2,980 m. x 4,530 m., vers 1660 (Versailles, Château de Versailles, MV6165, cliché : 04-510998). 
forebears ${ }^{61}$. " Cette composition de Le Brun est connue et largement diffusée dans l'Europe des Lumières, en particulier grâce aux gravures qui en sont produites. En outre, André Félibien y consacre un ouvrage qui sera traduit en 1703 sous le titre: The Tent of Darius Explain'd ${ }^{62}$. L'œuvre exprime toute la grandeur d'âme d'un souverain au faite de sa puissance, mais qui sait mesurer sa victoire en soulageant les victimes. Félibien, s'adressant à Louis XIV, lui décrit ainsi l'œuvre : "C'est savoir regner, Sire, que d'avoir remporté tant de victoires, conquis tant de Villes, donné la paix à toute l'Europe, établi le repos dans son Royaume; que de travailler continuellement au soulagement de ses peuples, \& chercher soy-mesme les moyens de les rendre heureux [...]", avant d'évoquer spécifiquement ce qui est rendu dans la représentation : «[...] on y peut remarquer qu'Aléxandre venant de rassûrer la famille de Darius qui estoit dans la crainte $\&$ dans le desordre, il y a dans toutes les actions de ce Prince [...] un certain calme, $\&$ une tranquilité qui ne témoignent que du repos $\&$ de la douceur $^{63}$. " Pour Félibien comme pour tous les contemporains, l'Alexandre de Le Brun, c'est Louis XIV.

Cette représentation n'est pas la seule que l'on doit considérer, car la même scène, peinte par Paolo Veronese en 1565-1567 (voir l'illustration 9), montre un Alexandre beaucoup plus conquérant, sûr de son pouvoir. Il n'y a pas d'humanité dans le geste. Le peintre italien a choisi de représenter le moment où le sort des conquis n'est pas encore scellé. La tension se lit sur le visage de ceux-ci, tandis que

${ }^{61}$ Jonathan Conlin, «Benjamin West's General Johnson and Representations of British Imperial Identity, 1759-1770: An Empire of Mercy? ", Journal for EighteenthCentury Studies, vol. 27, n 1 (mars 2004), p. 43. Voir également Emma Baker, "From Charity to Bienfaisance: Picturing Good Deeds in Late Eighteenth-Century France ", Journal for Eighteenth-Century Studies, vol. 33, n 3 (septembre 2010), p. 285-311.

${ }^{62}$ André Félibien, The Tent of Darius Explain'd; or the Queens of Persia at the Feet of Alexander, traduit du français par William Parsons, Londres, W. Rogers and H. Rhodes, 1703.

${ }^{63}$ André Félibien, Recueill de descriptions de peintures et d'autres ouvrages faits pour le Roy, Paris, Marbre-Cramoisy, 1689, p. 26 et 51. 


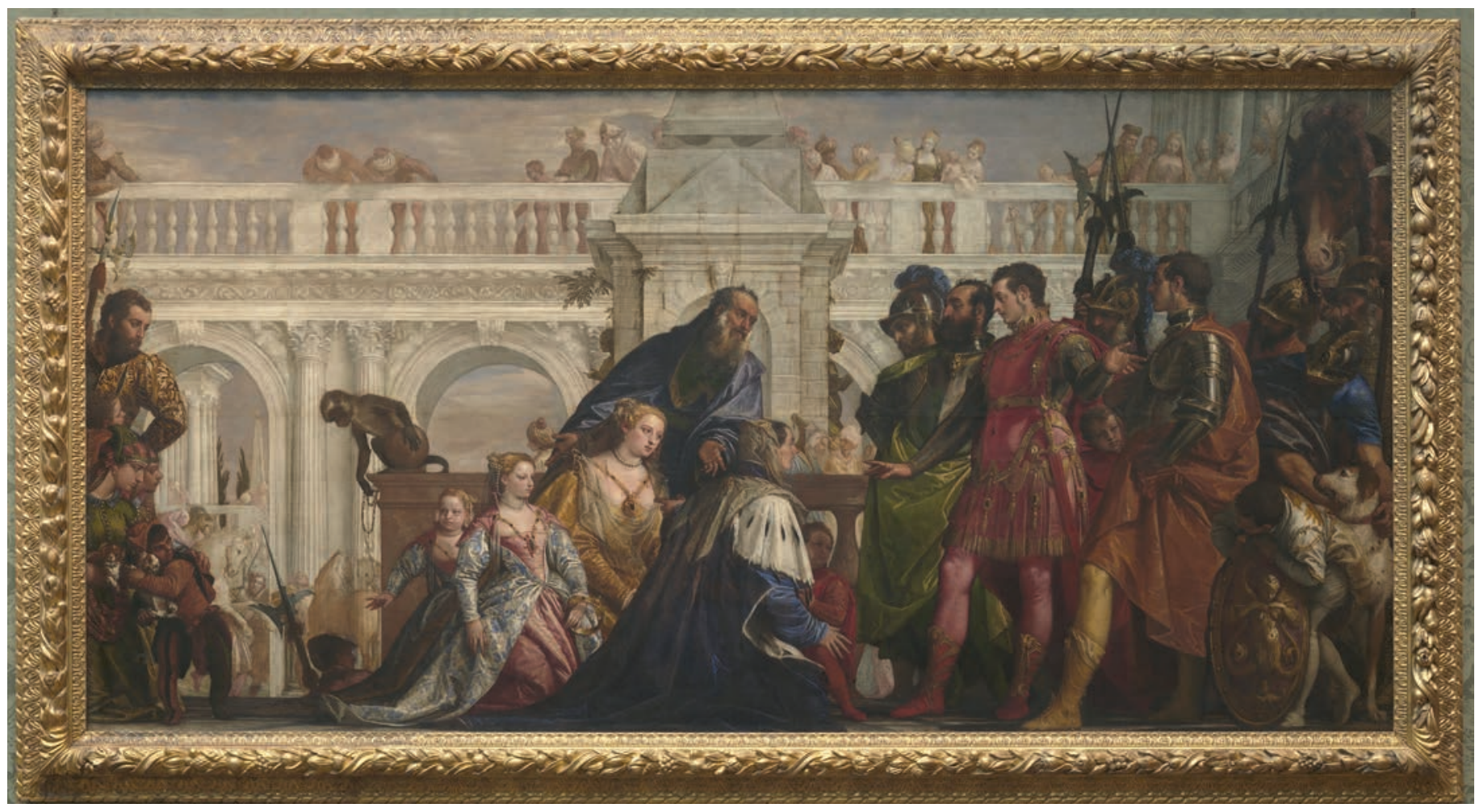

Ill. 9. Paolo Veronese (1528-1588), The Family of Darius before Alexander, huile sur toile, 236,2 cm x 474,9 cm, 1565-1567 (Londres, The National Gallery, nº NG294). 
l'Alexandre de Le Brun est déjà en train d'offrir sa mansuétude à la foule qui s'approche pour l'accueillir. Hayman reprend donc un thème connu, mais en introduisant quelques variations particulièrement intéressantes. Chez Le Brun, Alexandre est sans conteste associé à Louis XIV, roi de guerre par excellence ${ }^{64}$, tandis que Francis Hayman remplace la figure royale par celle d'un général. Certes, on assiste au $\mathrm{XvIII}^{\mathrm{e}}$ siècle à la glorification d'officiers militaires, distincte de celle de la figure royale, mais dans le présent contexte, ce n'est plus le souverain dont on veut faire l'éloge par l'entremise de la représentation d'Amherst, mais le patriotisme britannique.

Il est intéressant de noter que The Royal Magazine publie, en novembre 1759, une lettre d'un certain H. L., qui soutient que l'acte d'Alexandre n'est pas si méritoire puisque " every private man, and even a ruffian' would do the same if placed in a similar situation ${ }^{65}$ ". C'est précisément dans ce contexte que s'inscrivent l'humanité, la clémence et la charité du général Amherst. Ces valeurs, tout antiques qu'elles soient, ne sont pas réservées qu'aux grands; elles montrent, au contraire, par la confiance que manifeste l'homme envers ses semblables, sa capacité à ouvrir les bras, à proposer la concorde plutôt que la guerre. C'est ce que représente Francis Hayman. Il convient non seulement de représenter ces valeurs dans le contexte contemporain, mais aussi de permettre à tous les sujets de s'emparer de ces valeurs et de les faire siennes. Le texte et l'image ont pour fonction de remettre entre les mains du spectateur la gloire d'Amherst. Fier de la victoire anglaise, le spectateur peut se féliciter de faire partie d'une nation aussi ouverte et à laquelle il participe activement en admirant le talent d'Amherst et de Hayman. Humanité, charité et clémence doivent devenir des valeurs typiquement anglaises. Hayman, par l'entremise de Jonathan Tyers, participe à ce mouvement qui saisit l'Angleterre.

${ }^{64}$ Joël Cornette, Le roi de guerre : essai sur la souveraineté dans la France du Grand Siècle, Paris, Payot, 1993.

${ }^{65}$ The Royal Magazine, or, Gentleman's Monthly Companion, novembre 1759, Londres, Printed, pursuant to His Majesty's Royal Licence, for J. Coote, at the King's Arms in Pater Noster Row, 1759, p. 294-295. 
Les référents antiques dans les compositions de Hayman sont évidents. Entre la tente à la romaine, l'obélisque ou encore le mouvement du corps d'Amherst, rappelant celui d'Alexandre dans la composition de Le Brun, nous nous trouvons ici à mi-chemin entre la peinture d'histoire et la scène de bataille. Pourtant, ce n'est pas l'armée que Hayman a choisi de représenter ou encore la capitulation finale. À aucun moment n'apparaissent clairement les officiers français; on ne voit que la population montréalaise, les nouveaux sujets à qui il faut offrir la bienveillance anglaise. La France brille par son absence, si ce n'est le drapeau fleurdelisé qui est à peine visible. Hayman représente l'horizon d'attente des Britanniques avec cette victoire, horizon, comme nous l'avons vu dans les journaux, qui intègre le commerce et la paix. La supériorité britannique, supériorité des valeurs et des mœurs, a assuré la victoire.

Les valeurs d'Amherst, bien qu'elles soient inspirées de l'Antiquité, sont adaptées au goût du jour. Elles ont pour fonction de légitimer les conquêtes, de montrer, comme le disait l'auteur de A Description of Vaux-hall Gardens en 1762, "that clemency is the Genius of the British Nation ${ }^{66}$ ". La clémence est une des vertus de la Rome classique les plus célébrées dans les textes de Sénèque, de Tite-Live et de Cicéron ${ }^{67}$. Dans le contexte nord-américain de la guerre de Sept Ans, l'Angleterre emprunte le chemin de la vertu, celui que la Rome antique avait tracé. Ces valeurs sont aussi celles de la religion chrétienne, valeurs qui sont intrinsèquement liées à la figure d'un Christ rédempteur, ouvert, celui-là même qui affirmait qu'il fallait laisser venir à lui les petits enfants. Amherst devient ce Christ qui accueille, bras ouverts, une population apeurée par tant de violence et de sang. Plutôt que d'appliquer une vengeance sans merci, il propose l'inclusion, l'ouverture. Ainsi, " his own humane disposition' preserved the citizenry from being scalped, restored their property and caused them

\footnotetext{
${ }^{66}$ A Description of Vaux-hall Gardens, p. 25.

${ }^{67}$ Voir Sabine Rochlitz, Das Bild Caesars in Ciceros Orationes Caesarianae: Untersuchungen zur clementia und sapientia Caesaris, Frankfurt, Peter Lang, 1993.
} 
to be $f e d^{68}$ ". Je pourrais ajouter qu'une autre figure chrétienne est réactivée, en 1749, dans la capitale britannique, celle du roi Salomon. En effet, c'est le 17 mars de cette même année que Georg Friedrich Haendel présente son oratorio Solomon au public londonien à Covent Garden $^{69}$. L'œuvre sera reprise en 1759, soit quelques jours avant la mort du compositeur. Si la sagesse est la caractéristique principale du roi Salomon, on peut aussi y joindre la mansuétude, notamment avec l'épisode des deux prostituées ${ }^{70}$. Haendel proposera d'ailleurs quelques pièces musicales aux promeneurs qui arpentent les allées du Vauxhall ${ }^{71}$. Bien sûr, le Christ et le roi Salomon ne constituent que des figures d'inspiration et non des références directes.

The Surrender of Montreal répond ici à tout un contexte qui vise à représenter les guerres en Amérique comme le prélude à un empire qui apportera le bien-être aux populations. Plusieurs auteurs de l'époque s'accordent pour dire que l'humanité, la clémence et la charité sont propres à la nation anglaise et qu'elles permettent à l'Angleterre d'aspirer à la monarchie universelle grâce à son empire colonial $^{72}$. John Brown, vicaire de Newcastle sur Tyne, dans Les Moeurs Angloises ou Appréciation des mours et des principes qui caractérisent actuellement la nation britannique (1758), œuvre traduite en français, affirme lui aussi que les Britanniques ont su développer cette humanité qui les différencie des autres peuples d'Europe :

${ }^{68}$ A Description of Vaux-hall Gardens, p. 25.

${ }^{69}$ Joël Richard, «Chant de guerre, chant de paix : les oratorios de Haendel de 1746 à 1749 ", dans Paul-Gabriel Boucé (dir.), Guerres et paix: la Grande-Bretagne au XVIII siècle, Paris, Presses de la Sorbonne nouvelle, 1999, vol. 2, p. 303-310.

${ }^{70}$ Les deux femmes se présentent devant le roi Salomon en soutenant être mère du même enfant. Salomon propose de trancher le bambin en deux pour en donner une partie à chacune. C'est alors que la vraie mère empêche le garde d'appliquer la sentence et affirme que la seconde femme peut partir avec l'enfant. Avec ce stratagème, le roi réussit à déterminer qui est véritablement la mère de l'enfant.

${ }^{71}$ Terence Hodgkinson, "Handel at Vauxhall », Victoria \& Albert Museum Bulletin, vol. 1, no 4 (octobre 1965), p. 1-12.

${ }^{72}$ Frances A. Yates, Astrée : le symbolisme impérial au XVe siècle, Paris, Belin, 1989; Lucien Bély, L'art de la paix en Europe : naissance de la diplomatique moderne, $X V I^{e}$-XVIIt siècles, Paris, Presses universitaires de France, 2007. 
L'Humanité dont je parle, est cette compassion tendre pour les malheureux, cette modération adroite à restreindre les peines qu'on inflige, par des adoucissemens tirés du but de ces peines $\&$ du degré qu'il convient de leur assigner; sentimens généreux qui ont toujours distingué la Nation Angloise entre toutes les autres.

La douceur de nos Loix contre les crimes capitaux ; notre compassion pour les plus insignes coupables; tout jusqu'aux procédés de nos voleurs de grand chemin, comparés à ceux des autres pays, annonce que l'esprit d'Humanité est spécifiquement propre aux Anglois.

[...] il faudroit [...] reconnoitre [à la Nation] son Humanité, d'y applaudir, \& de l'en estimer comme elle le mérite ${ }^{73}$.

En 1751, Hume, dans An Essay Concerning the Principles of Morals, affirmait déjà que " [a] patriot who serves our own country, in our own time, has always a more passionate regard paid to him, than one whose beneficial influence operated on distant ages or remote nations, where the good, resulting from his generous humanity, being less connected with us, seems more obscure, and affects us with a less lively sympathy ${ }^{74}$ ". Ces définitions sont données à un public britannique qui voit l'Empire s'agrandir et se définir. Il est donc nécessaire de légitimer les conquêtes, apporter la lumière de l'humanité, en exploitant une fibre patriotique impériale.

Un peu comme les Romains avec la Pax Romana, cette Pax Britannica est assurée, cette fois, par un socle de valeurs chrétiennes; l'identité de l'Empire britannique devient ouverture, humanité et charité. Une des caractéristiques principales de la Pax Romana était la capacité de l'Empire d'octroyer la citoyenneté romaine aux peuples nouvellement conquis, intégrant ainsi les ennemis passés au projet

${ }^{73}$ John Brown, Les Mours Angloises ou Appréciation des mours et des principes qui caractérisent actuellement la nation britannique, La Haye, Pierre Gosse, 1758, p. 13-14.

${ }^{74}$ David Hume, An Essay Concerning the Principles of Morals, éd. Tom L. Beauchamp, Cambridge, Cambridge University Press, 1998, p. 43. 
commun de domination universelle. C'est exactement ce que se propose de faire l'Empire britannique à la suite de la conquête du Canada puisque cette Pax Britannica doit elle aussi être capable d'intégrer ses nouveaux sujets. Pour y arriver, il faut présenter des valeurs d'ouverture, de concorde et d'humanité, ce que fait parfaitement bien Francis Hayman dans son tableau.

\section{Conclusion}

Il y a une adéquation entre ce qui est représenté dans le tableau de Hayman et ce qui est compris par les contemporains, car même les Français arrivent à voir l'aspect civilisateur de l'œuvre. L'identité impériale britannique ne peut se définir uniquement contre la France; elle possède des caractéristiques universelles qui dépassent ces conflits. L'œuvre de Hayman permet de comprendre les multiples facettes de cette identité en apportant un éclairage supplémentaire sur la culture visuelle londonienne touchant la guerre de Sept Ans. Le présent texte a également permis de s'interroger sur la manière dont a été perçue la conquête du Canada par les Britanniques et d'analyser comment celle-ci a été insérée dans la trame plus grande de la construction de l'Empire.

Pourtant, l'histoire de la prise en charge et de l'imposition du modèle britannique au Canada est loin d'être aussi harmonieuse que ce qui est présenté dans l'œuvre ici étudiée. La religion catholique est déjà bien établie, les valeurs ne sont pas toutes les mêmes et, très vite, les nouveaux maîtres du Canada devront s'adapter au système de justice, à la langue ainsi qu'aux habitudes des nouveaux sujets britanniques. Reste cependant qu'avec des valeurs d'humanité, de charité et de clémence, il devient difficile de légitimer de violents coups de force contre une population nouvellement conquise. Il faut donc se rabattre sur le compromis et le respect de l'autre. L'idée est alors d'intégrer ces peuples dans le projet de construction de l'empire. Il faut imposer la Pax Britannica aux nouveaux sujets de Sa Majesté 
afin qu'ils puissent profiter de ce que l'on considère comme la supériorité culturelle de l'Empire britannique.

La suite des événements est loin d'avoir confirmé cette version des faits ${ }^{75}$. L'œuvre de Francis Hayman permet de saisir le travail de persuasion réalisé dans l'Angleterre de la seconde moitié du siècle pour faire accepter les conquêtes, en en montrant les avantages. Une idée que résume particulièrement bien le Français Grosley, en 1774, quand il évoque un dialogue qu'il a eu avec un Londonien :

[...] pour lui couper chemin, je lui montrai mon chapeau, acheté la veille à Londres $\&$ taillé à l'Angloise. Ne se le tenant pas pour dit, il ajouta que c'étoit uniquement par goût qu'il se coëffoit ainsi, qu'il n'y étoit point forcé par la nécessité; \& vint la conquête du Canada. C'est apparemment, lui dis-je, cette conquête qui vous a diminué le prix de la bière $\&$ qui vous a établi le pain à si bon marché (le pain valoit alors 7 sols, $\&$ au moyen d'un impôt occasionné par la derniere guerre $\&$ non supprimé, la bière étoit augmentée de $2 \mathrm{~s}$. par pinte). Vous entendez les affaires, répondit l'Anglois; mais toujours est-il vrai que nous avons de notre côté le Canada \& le Castor. Avant, lui répliquai-je, que d'entrer plus avant dans cette matière, dites-moi ce que vous avez gagné personnellement à cette conquête; $\&$ je vous dirai ce que j'y perds personnellement : il sourit, \& revint de très-bonne grace à m'expliquer la tragédie que l'on jouoit ${ }^{76}$.

L'important est de tirer des avantages d'une conquête ou, à tout le moins, de montrer au public que la grandeur d'âme britannique est doublée de bénéfices commerciaux.

${ }^{75}$ Voir notamment José E. Igartua, « A Change in Climate: The Conquest and the Marchands of Montréal ", Historical Papers = Communications historiques, vol. 9, $\mathrm{n}^{\circ} 1$ (1974), p. 115-135; Donald Fyson, «The Canadians and British Institutions of Local Governance in Quebec, from the Conquest to the Rebellions ", dans Nancy Christie (dir.), Transatlantic Subjects: Ideas, Institutions, and Social Experience in Post-Revolutionary British North America, Montréal, McGill-Queen's University Press, 2008, p. 45-82.

${ }^{76}$ Grosley, Londres, p. 176-177. 